determination of the area of developed grains will give slightly higher values than those obtained from electron micrographs of unexposed grains. This fact should be taken into account when the grain size is determined from photometric measurements.

We have dealt with Q2-plates ( Il f o r d Ltd.) up to now. Some measurements have been carried out with Q1-plates (Ilford Ltd.) an electron micrograph of which is shown in Fig. 8.
The fraction of the area covered with visible grains was found to be 0.73 , the average projection area being smaller than for Q2-plates.

\section{Acknowledgement}

The authors wish to thank Mr. RoEPERs for doing much of the numerical work and Mr. Schelling for the measurement of the emulsion thickness.

\title{
Ladungsträgerverluste in einem schwachionisierten stationären Gleichstromplasma im toroidalen Magnetfeld
}

\author{
F. KARGER \\ Max-Planck-Institut für Physik und Astrophysik, München \\ (Z. Naturforschg. 22 a, 1039-1057 [1967] ; eingegangen am 14. März 1967)
}

\begin{abstract}
For the particle losses of a weakly ionized plasma which result from the torus drift in a curved magnetic field, an expression is derived which is valid for certain parameters of the positive column of a gas discharge. To check this theory the "AMBIPOL" device was built. With this device it was possible to determine simultaneously the losses both in the toroidal and in the linear magnetic field by measuring the longitudinal electric field strength. As theory predicts, with growing magnetic field strength a weaker decrease of the longitudinal electric field was observed in the toroidal part of the discharge as compared to the linear part. The measured values of the relative electric field strength, however, exceed the theoretical limit, although the measurements of the electric field in the straight part and the measurements of the particle density and of the electron temperature in the curved part are consistent with theory. Moreover, contrary to the expectations, the onset of the KaDomTSEV instability occurs at lower critical magnetic fields in the toroidal part than in the straight part. Several possible explanations are discussed. In a later paper it will be attempted to make a choice among the three most probable ones.
\end{abstract}

\section{A. Problemstellung und qualitativer Überblick}

Der noch immer nicht völlig geklärte „Pump out“. Prozeß in Plasmamaschinen vom Typ des STELLARATORs gab den Anlaß, in einem Modell-Plasma den Einfluß der Krümmung des Entladungsrohres und der Magnetfeldlinien auf den Teilchenverlustmechanismus zu untersuchen. Als ein ohne allzu großen Aufwand herstellbares Laboratoriums-Plasma bot sich dabei die positive Säule einer stationären Niederdruck-Gasentladung mit nichtleitenden Wänden an.

In der Apparatur AMBIPOL (Abb. 1) wird die positive Säule mit überlagertem longitudinalen Magnetfeld in einen linearen (L) und einen toroidalen Anteil (T) aufgeteilt. (In den Abbildungen sind die Bezeichnungen $\mathbf{L}, \mathbf{T}$ jeweils in einen Kreis gesetzt.) Die dadurch bedingte Gleichheit von Neutralgasdruck und Entladungsstrom in beiden Teilen erscheint als gute Basis für einen Vergleich zwischen

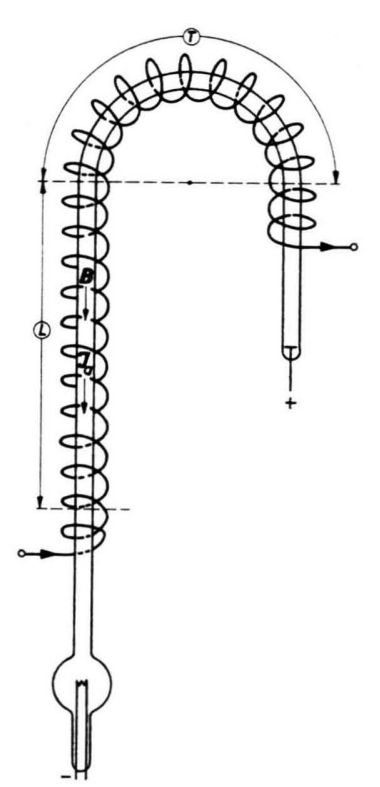

Abb. 1. Schema von AMBIPOL. 
$\mathbf{L}$ und $\mathbf{T}$, da sich die interessierenden Entladungsparameter in der gleichförmigen positiven Säule bei einer Translation parallel zur Entladungsröhre nur vernachlässigbar ändern (Abb. 2).

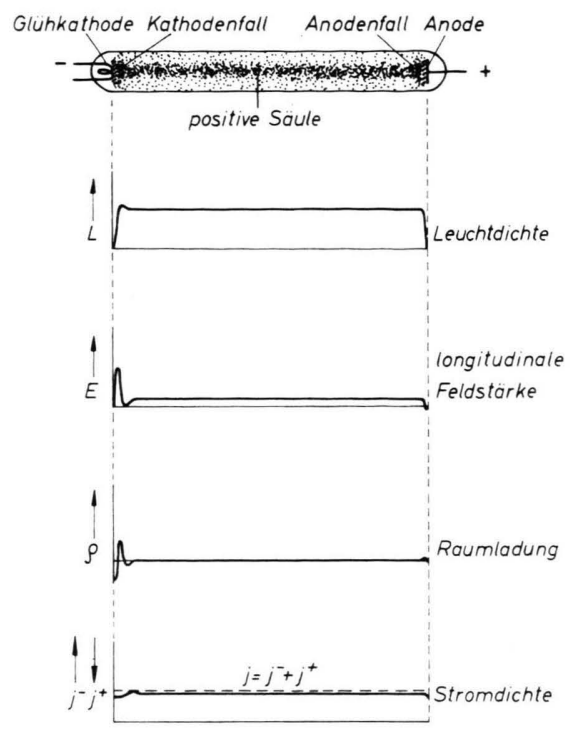

Abb. 2. Schematischer Verlauf der Entladungsparameter längs der Achse einer Glühkathodengasentladung.

In einem qualitativen Überblick können wir uns klarmachen, daß in der stabilen positiven Säule die Teilchenverluste im zylindrischen Teil (L) mit steigendem Magnetfeld wegen der Verringerung der ambipolaren Diffusion quer zum Magnetfeld abnehmen werden (vgl. ${ }^{1}$ ). Demgegenüber werden im toroidalen Teil auf Grund des Magnetfeldgradienten $\nabla B$ die verschiedenen Ladungsträger eine Drift

$\odot \boldsymbol{B}$ (aus der Ebene herous nach oben)

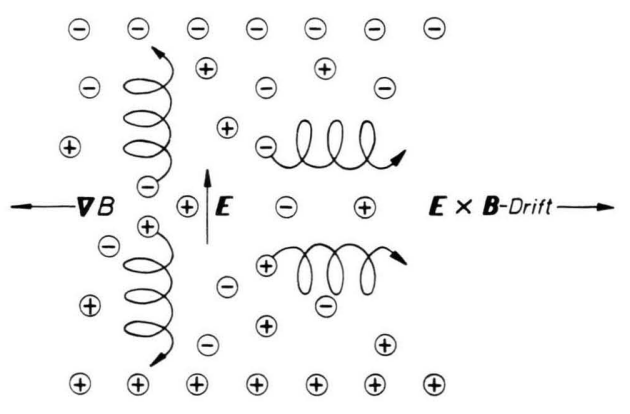

Abb. 3. Torusdrift (schematisch).

1 R. J. Bickerton u. A. v. Engel, Proc. Phys. Soc. B 69, 468 1956, S. 5.

2 L. Spitzer Jr., Physics of Fully Ionized Gases, New York 1956, S. 5. nach entgegengesetzten Richtungen senkrecht zum Magnetfeld und Magnetfeldgradienten erfahren (Abb. 3, vgl. etwa ${ }^{2}$ ). Das dadurch erzeugte Raumladungsfeld $\boldsymbol{E}$ bewirkt eine (ambipolare) $\boldsymbol{E} \times \boldsymbol{B}$. Drift, die der Richtung des Magnetfeldgradienten entgegengesetzt ist. Der dadurch hervorgerufene Anteil der Verluste wird also mit wachsendem Magnetfeld ansteigen.

An Hand eines vereinfachten Bildes der Entladung können wir uns ferner veranschaulichen, daß die positive Säule nicht für alle Magnetfeldstärken stabil sein kann auf Grund einer von Kadomtsev und Nedospasov ${ }^{3,4}$ gedeuteten konvektiven Instabilität (im weiteren K-Instabilität genannt). Dazu betrachten wir den Verlauf einer zufälligen schraubenförmigen Störung des Entladungskanals (Abb. 4). Ist kein Magnetfeld vorhanden, so wird jede eintretende Störung des Entladungskanals, d. h. jede seitliche

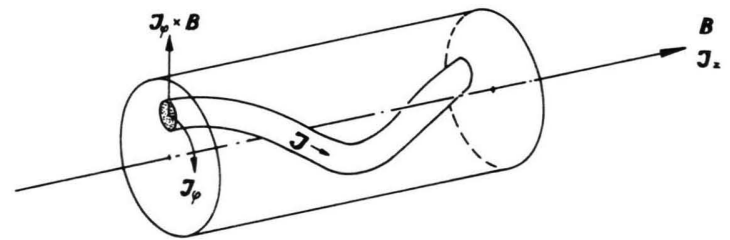

Abb. 4. Schraubenförmige Störung des Entladungskanals.

Verschiebung der radialen Ladungsträgerdichteverteilung durch eine aus der Veränderung des Dichtegradienten resultierenden Vergrößerung bzw. Verkleinerung der Diffusion zur Wand wieder zurückgebildet. Bei Anwesenheit eines longitudinalen Magnetfeldes $\boldsymbol{B}$ kann jedoch die Azimutal-Komponente $\boldsymbol{I}_{\varphi}$ des gestörten Stromes eine $\boldsymbol{I}_{\varphi} \times \boldsymbol{B}$-Kraft nach außen ergeben, falls etwa bei einer rechtsgängigen Schraube das Magnetfeld $\boldsymbol{B}$ und der Hauptstrom $\boldsymbol{I}_{z}$ gleichsinnig parallel sind. Da der ambipolare Diffusionskoeffizient mit steigendem Magnetfeld sinkt (s. o.), kann bei genügend hohen magnetischen Feldstärken die Diffusion die $\boldsymbol{I}_{\varphi} \times \boldsymbol{B}$-Kraft nicht mehr kompensieren, der Entladungskanal wird an die Rohrwand gedrängt und es entsteht eine turbulente Entladung. Dies bedeutet eine Erhöhung der Verluste mit dem Magnetfeld oberhalb einer bestimmten kritischen Magnetfeldstärke $B_{\mathrm{c}}$ im geraden und gekrümmten Teil der Entladung.

${ }^{3}$ B. B. Kadomtsev u. A. V. Nedospasov, J. Nucl. Energy, Part C, 1, 230 [1961].

${ }^{4}$ F. Karger, Seminar über Fragen der Plasmaphysik, MPI f. Phys. u. Astrophys. (4-1963). 
Die Frage lautet nun, welcher der drei Verlustmechanismen der jeweils vorherrschende sein wird und wie sie sich gegenseitig beeinflussen werden. Durch diese Fragestellung ist die experimentelle Anordnung in ihren Grundzügen festgelegt.

\section{B. Experimentelle Anordnung von AMBIPOL}

\section{Aufbau der Apparatur}

\section{a) Vakuumsystem}

In Abb. 5 ist das Schema des Hochvakuum-Systems von AMBIPOL gezeigt. Das eigentliche Entladungsgefäß besteht aus einem 4-teiligen Pyrex-Glasrohr von $380 \mathrm{~cm}$ Gesamtlänge und 5,2 cm innerem Durchmesser (vgl. auch Abb. 6). Der Krümmungsradius der Torusseele beträgt $35 \mathrm{~cm}$. Die Entladungsröhre wird an beiden Seiten über 3 Hochvakuum-Glashähne mit $35 \mathrm{~mm}$ Bohrung mittels zweier baffle-geschützter Öldiffusionspumpen (LEYBOLD DO 121 als Hochvakuum-Pumpe, DO 30 als Vorstufe) und einer zweistufigen Vorpumpe (D 6) evakuiert. Der Basisdruck liegt bei $10^{-6}$ Torr.
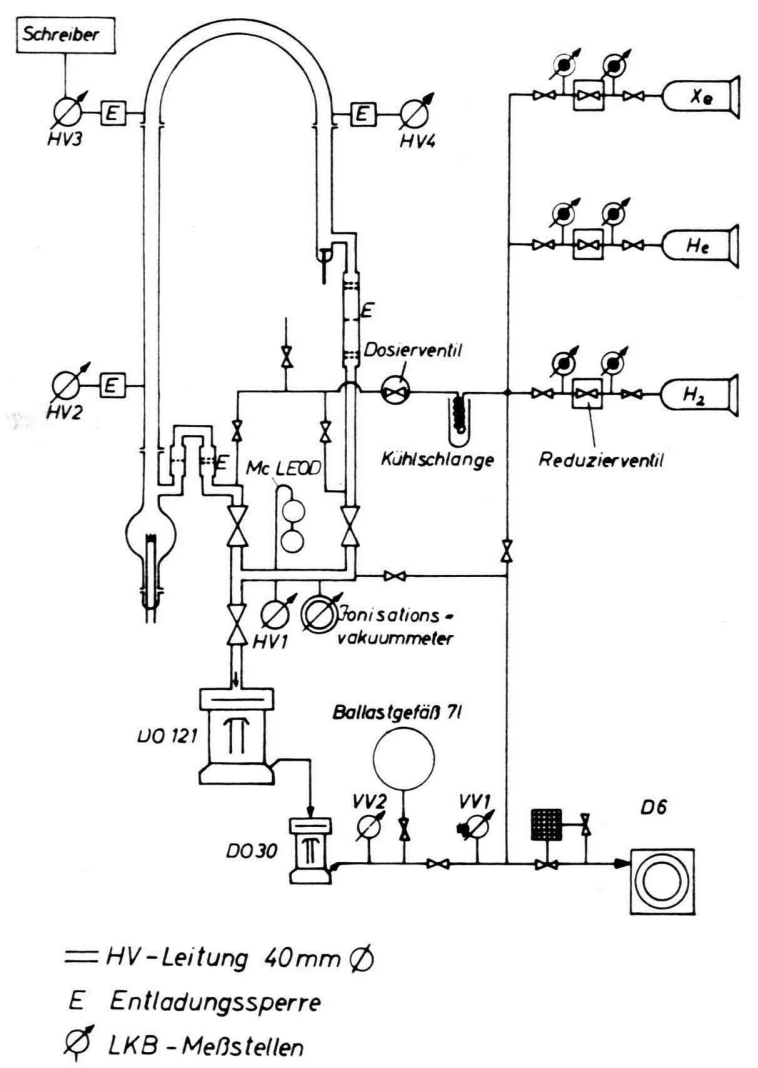

Abb. 5. Vakuumsystem.

5 H. Franke u. H. BaUer, Vakuumtechnik 14, 185 [1965].

${ }^{6}$ F. KARGER, Vakuumtechnik 13, 152 [1964].
Aus Reinheitsgründen wird mit durchströmendem Neutralgas gearbeitet. Dabei gelangt das jeweilige nachgereinigte $\mathrm{Gas}\left[\mathrm{H}_{2}(99,999 \%), \mathrm{He}(99,995 \%)\right.$ und $\mathrm{Xe}$ $(99,9 \%)]$ aus der Stahlflasche über ein Reduzierventil, eine Kühlschlange in flüssigem Stickstoff (außer bei Xenon) und ein Dosierventil auf ein Hahnsystem, das den Gasdurchsatz durch die Entladungsröhre wahlweise in beiden Richtungen gestattet. Der Arbeitsdruck $\left(10^{-2}\right.$ bis 3 Torr) wird an einem LKB-Pirani-Manometer abgelesen, das mittels eines McLeodschen Manometers geeicht wird. Mit Hilfe von 3 verschiedenen thermisch abgeschirmten Pirani-Manometerröhren am Entladungsrohr ist der Druckgradient entlang des Entladungsrohres feststellbar. Da der Einsatzpunkt der K-Instabilität druckabhängig ist, mußte mit möglichst niedrigem Druckgradienten gearbeitet werden. Die maximale Druckdifferenz zwischen HV 2 und HV 4 lag im ungünstigsten Falle (Xenon $10^{-2}$ Torr) bei $10 \%$. Bei den übrigen Gasen war sie nicht mehr meßbar.

Der Gasdurchsatz beträgt bei allen Experimenten ca. 0,3 Torr $\cdot 1 /$ sec. Beim Abpumpen dieser Gasmengen mit Öldiffusionspumpen stellen sich hochvakuumseitig periodische Druckschwankungen ein, die aus periodischen Änderungen der Lage des Verdichtungsstoßes resultieren (vgl. ${ }^{5}$ ). Durch geringes Überheizen der Vorstufe (DO 30) wurde eine stationäre Strömung erreicht.

Zur Verhinderung eines Durchschlags der Entladung von der Anode bzw. Kathode durch die relativ weiten Absaugrohre zur Pumpe wurde eine Entladungssperre entwickelt ${ }^{6}$, die nach dem Prinzip der Hrtronfschen Umwegröhre arbeitet und auch zur elektrischen Abtrennung der Pirani-Manometerröhren von der Entladung benutzt wurde.

Da bestimmte Verhaltensweisen der K-Instabilität empfindlich von Verunreinigungen abhängen ${ }^{7}$, werden vor dem jeweiligen Experiment

1. alle Gas-Zuführungsteile vom Abschlußhahn der Stahlflasche bis zum Dosierventil auf Vorvakuum evakuiert,

2. alle HV-Leitungen elektrisch ausgeheizt,

3. durch Ausheizen der Sauereisenmasse der Entladungssperren eine zusätzliche Getterwirkung erreicht,

4. zur Ausheizung der Entladungsröhre die Entladung in dem jeweiligen Gas bei einem Strom von ca. $1 \mathrm{~A}$ ein paar Stunden gebrannt.

Die Reinheit des jeweiligen Entladungsplasmas wird spektroskopisch geprüft.

\section{b) Magnetfeld}

Das zur Entladungsachse parallele Magnetfeld wird von 4 wassergekühlten Spulen erzeugt, die eine Gesamtlänge von $260 \mathrm{~cm}$ und einen inneren Durchmesser von $10 \mathrm{~cm}$ besitzen (Abb. 6). An die Magnetfeldspulen müssen bezüglich ihrer Homogenität hohe Anforderun-

7 T. SeKIGUCHI, persönl. Mitteilung. 


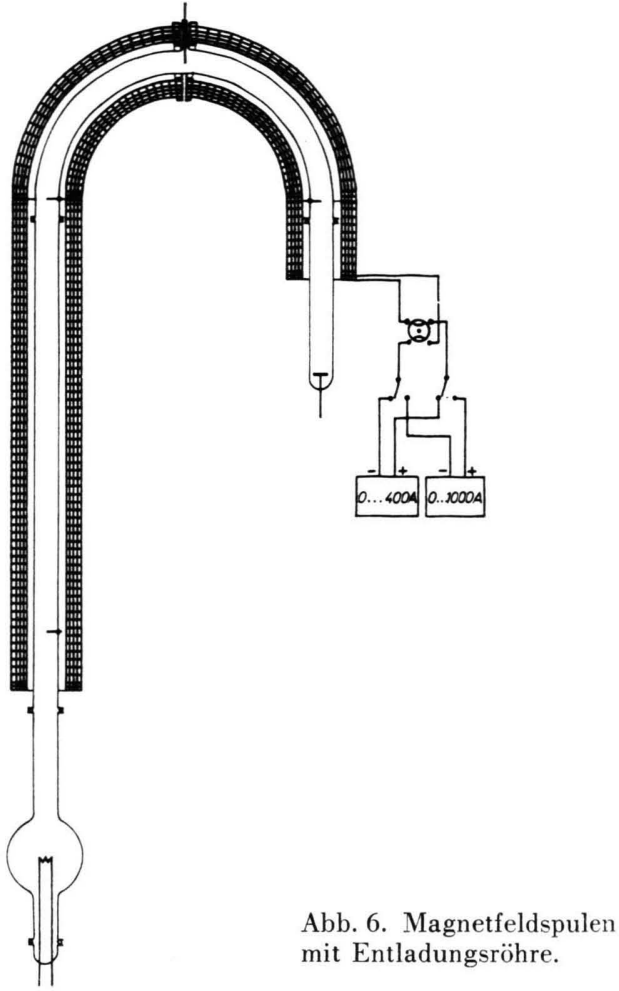

gen gestellt werden. Denn im Falle einer inhomogenen, bezüglich der Achse mit der Periodenlänge $l$ periodischen Magnetfeldstärke mit den Extremwerten $B_{\text {min }}$ und $B_{\max }$ überwiegt im Plasma bei höheren Neutralgasdrucken $p(>0,5$ Torr $)$ die transversale Diffusion $\left(D_{\perp}\right)$ die longitudinale Diffusion $\left(D_{\|}\right)$, d. h.

$$
l^{2} / D_{\|} \pi^{2} \gg \Lambda_{0}^{2} / D_{\perp}
$$

( $D_{\|}, D_{\perp}$ Diffusionskoeffizienten, $A_{0}$ Diffusionslänge). In diesem Fall ist die Diffusion durch $B_{\max }$ bestimmt. Wenn sich die obige Relation jedoch umkehrt (bei Drucken $<0,05$ Torr), ist der kürzeste Diffusionsweg von einem Gebiet mit maximaler magnetischer Feldstärke: erst longitudinale Diffusion in ein $B_{\min }$-Gebiet mit anschließender transversaler Diffusion. Hier ist die Diffusion also durch $B_{\min }$ bestimmt.

Um den dadurch bedingten systematischen Fehler zu vermeiden, wurde jede der 4 Spulen in einem Stück gegensinnig in 4 Lagen durchgehend gewickelt. Dadurch und durch ein System von Hilfsspulen an den Spulenenden und variablen Shunts wurde die auf der Achse gemessene Inhomogenität auf der gesamten in Betracht kommenden Länge auf $1 \%$ gedrückt. Sowohl die toroidalen als auch die zylindrischen Spulen wurden zunächst dicht gewickelt und dann durch Abstandsstücke in ihre endgültige Form gebracht und mit Epoxydharz vergossen. Dieses Verfahren hatte sich trotz

${ }^{3}$ K. Moustafa, Techn. Ber. d. Elektronikgruppe des MPI f. Phys. u. Astrophys. 6-65-40.1 [1965]. der Notwendigkeit umfangreicher technologischer Voruntersuchungen über das Elastizitätsverhalten von Kupfer (kontrolliertes „Aufgehen“ der Windungen) als das einfachste und beste bewährt. Die Abstandsstücke konnten nach dem Vergießen wieder entfernt werden, wodurch Raum für die optische Beobachtung der Plasmasäule gewonnen wurde.

Als Stromquellen wurden ein Dreiphasen-40 V/400 AGleichrichter und eine $100 \mathrm{~V} / 1000 \mathrm{~A}$-Maschine benutzt. Damit konnten im stationären Betrieb Magnetfeldstärken bis 1,7 kGauss und im langsamen Pulsbetrieb ( $1 \mathrm{sec}$, mit $10 \mathrm{sec}$ Intervall) bis zu 4,5 kGauss erreicht werden.

Die bei Pulsbetrieb in der Entladung induzierte elektrische Spannung war vernachlässigbar klein (ca. $1 \mathrm{mV})$, so daß hierbei die Quasistationarität gewahrt blieb.

\section{Entladung mit Stromversorgung}

Die Entladung (Abb. 7) wird durch Vorionisation mit Hilfe eines TESLA-Transformators (T) in Kathodennähe von außen gezündet. Als Stromquelle für die Entladung steht ein erdfreier Dreiphasengleichrichter $(3 \mathrm{kV} / 2 \mathrm{~A})$ zur Verfügung. Da die Erdung der Entladung zur Erreichung einer höheren Genauigkeit bei den Sondenmessungen kontinuierlich zwischen Anoden- und Kathodenpotential variabel sein muß $\left(R_{3}\right.$ bzw. $\left.R_{4}\right)$, wird eine doppelseitige erdfreie Siebung benötigt $\left(\mathrm{C}_{1}\right.$, $\left.\mathrm{L}_{1}, \mathrm{~L}_{2}, \mathrm{C}_{2}\right)$. Die Siebung der Brummspannung (300 $\mathrm{Hz}$ ) gegen Erde erfolgt symmetrisch im positiven und negativen Kanal durch je ein L-Glied $\left(\mathrm{L}_{1}, \mathrm{C}_{3}\right.$ und $\mathrm{L}_{2}$, $\mathrm{C}_{4}$ bzw. $\mathrm{L}_{1}, \mathrm{C}_{5}$ und $\mathrm{L}_{2}, \mathrm{C}_{6}$ ). Die Zeitkonstante der symmetrischen Erdung ist durch die Größe der Potentiometer $\mathrm{R}_{3}$ bzw. $\mathrm{R}_{4}$ und den zugehörigen Kondensatoren $\mathrm{C}_{3}, \mathrm{C}_{4}$ bzw. $\mathrm{C}_{5}, \mathrm{C}_{6}$ bestimmt $(\tau=R \cdot C=0,3 \mathrm{sec})$.

$\mathrm{Da}$ die für die Trägerverluste repräsentative, in der positiven Säule umgesetzte elektrische Leistung durch Differenzspannungsmessung an Sonden festgestellt wird, muß der Entladungsstrom $I_{\mathrm{a}}$ innerhalb eines bestimmten Spannungsintervalls $U_{\mathrm{D}}$ sehr genau konstant gehalten werden. Zu diesem Zweck wurde als Vorwiderstand für den Entladungskreis ein elektronischer Stromkonstanthalter $\mathrm{K}$ konstruiert ${ }^{8}$. Dieser Regler soll die Spannungsänderung aufnehmen, die bei Änderung der magnetischen Feldstärke über der Entladung entsteht, so daß die interessierenden Größen in Abhängigkeit vom Magnetfeld bei konstantem Strom direkt registriert werden können (vgl. $\left.{ }^{9}\right)$.

In einem Spannungsbereich von $U_{\mathrm{D}}=400 \mathrm{~V}$ beträgt die relative Stromänderung $\left(\Delta I_{\mathrm{a}} / I_{\mathrm{a}}\right) / \Delta U_{\mathrm{D}}=3 \cdot 10^{-5} / \mathrm{V}$. Der konstant zu haltende Strom ist von $100 \mathrm{~mA}$ bis 1,5 A kontinuierlich einstellbar. Durch das Erdungspotentiometer $\mathrm{R}_{4}$ und die Widerstände der Entladungssperren wird die Stromkonstanz nur innerhalb der Toleranzgrenzen verfälscht.

Die direkt geheizte Oxydkathode kann wahlweise an einen hochspannungsisolierten Transformator $(4 \mathrm{~V}$ /40 A) oder einen Akkumulator angeschlossen werden.

\footnotetext{
${ }^{9}$ K. H. Woehler, Z. Naturforschg. 17 a, 937 [1962].
} 


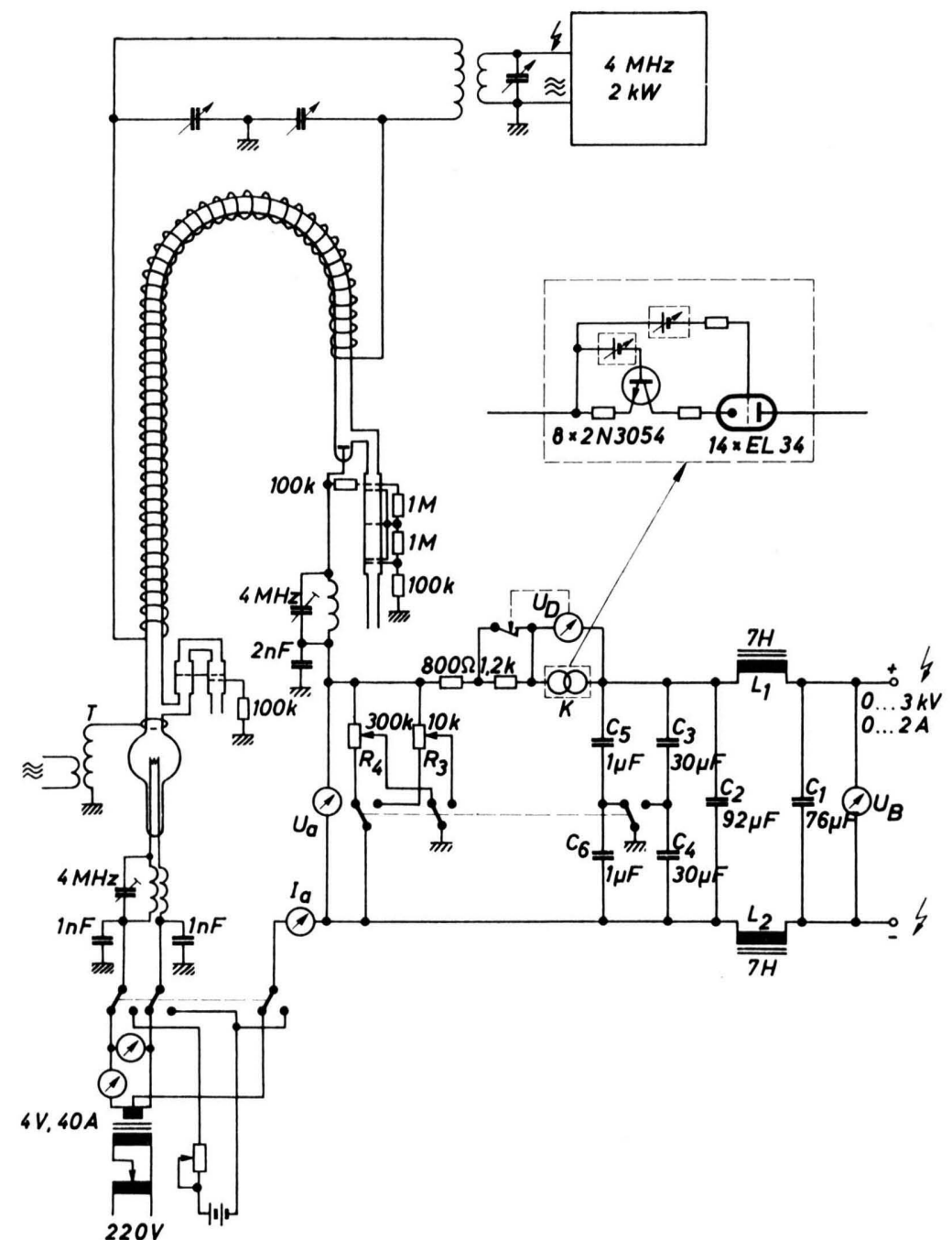

Abb. 7. Stromversorgung der Entladung.

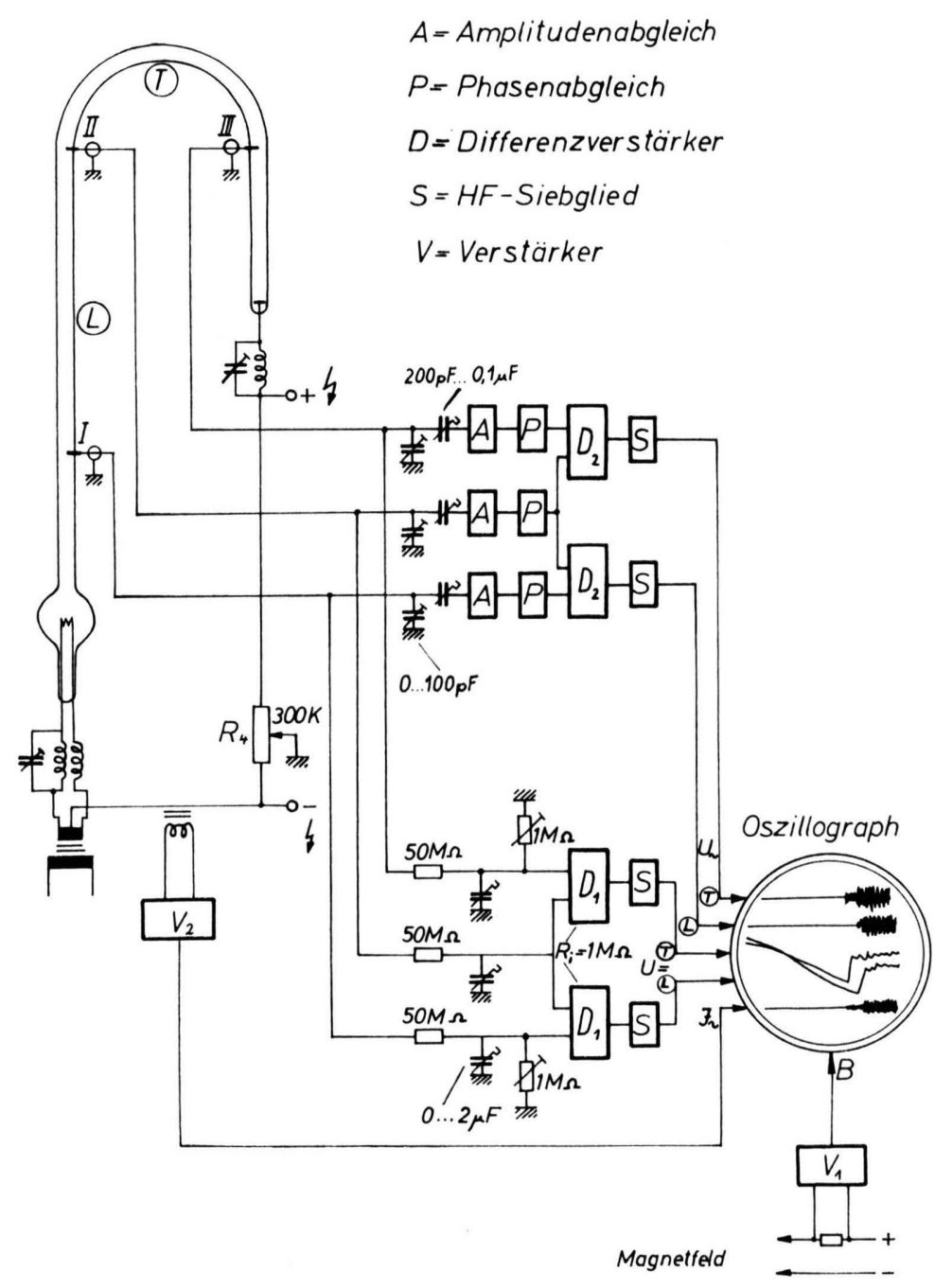

Abb. 8. Messung der longitudinalen elektrischen Feldstärke. 
Um die Ladungsträgererzeugung, die von den im longitudinalen elektrischen Gleichfeld beschleunigten Elektronen übernommen wird, teilweise durch eine Ionisierung im Hochfrequenzfeld zu ersetzen und dadurch das erforderliche elektrische Gleichfeld reduzieren zu können, wird das ganze Entladungsrohr mit einer Spule von $20 \mathrm{Wdg}$./m umgeben, die induktiv über einen Luftübertrager an einen HF-Sender (4 MHz, $0-2 \mathrm{~kW})$ angekoppelt wird. Die mit dieser Anordnung in das Plasma induktiv und kapazitiv einkoppelbare Leistung reicht aus, die gesamten Trägerverluste der Entladung durch HF-Ionisation zu decken. Zur Reduzierung des Gleichrichtereffektes an den Sonden ${ }^{10}$ wird die Plasmakopplungsspule kapazitiv symmetrisch geerdet.

\section{Diagnostikverfahren}

\section{a) Messung der longitudinalen elektrischen Feldstärke}

Als Maß für die Ladungsträgerverluste dient die elektrische Längsfeldstärke, die der in das betrachtete Volumen der positiven Säule eingespeisten elektrischen Leistung proportional ist. Die elektrische Feldstärke im zylindrischen und toroidalen Teil wurde bestimmt aus den Potentialdifferenzen zwischen drei Zylindersonden, die auf der Rohrachse in jeweils $110 \mathrm{~cm}$ Abstand voneinander angeordnet sind und sich auf Floatingpotential befinden (Abb.8). Die Sondenpotentiale werden dabei über einen 100 : 1-Spannungsteiler auf je einen Gleichspannungs-Differenzverstärker $\mathrm{D}_{1}$ und deren Ausgangssignal über HF-Siebglieder auf die $y$-Ablenkung eines Oszillographen gegeben.

Eine direkte Siebung der Sondenspannungen mit einer Zeitkonstante von $1 \mathrm{~ms}$ bis $1 \mathrm{~s}$ ist vorgesehen. Die $x$-Ablenkung erfolgt jeweils mit einem dem Magnetfeld proportionalen Signal, das an einem Serienwiderstand im Magnetfeldstromkreis abgegriffen und über $V_{1}$ verstärkt wird, so daß die zwei Potentialdifferenzen vom zylindrischen und toroidalen Teil $\left(U_{\mathbf{L}}\right.$ und $\left.U_{\mathbf{T}}\right)$ als Funktion des Magnetfeldes registriert werden können (vgl. Abb. 9). Dadurch, daß am Anfang jeder Messung für $B=0$ mit Hilfe von $\mathrm{R}_{4}$ Sonde II auf Erdpotential gebracht wurde, ergab sich ein minimaler Differenzenfehler und die Strombelastung der Sonden durch die $50 \mathrm{M} \Omega$-Widerstände der Spannungsteiler konnte unter $6 \mu \mathrm{A}$ gehalten werden $(50 \mathrm{M} \Omega$ ist genügend groß gegen den Plasmainnenwiderstand von ca. 100 bis 500 $\mathrm{k} \Omega$, so daß die Sonden als praktisch stromlos gelten können). $\mathrm{ab}$

Die Genauigkeit der Methode hängt im wesentlichen

1. von der Genauigkeit, mit der bei Variation des Magnetfeldes die Grenzschicht-Potentialdifferenzen von 2 Sonden gleich sind (vgl. Sondentheorie ${ }^{11}$ ),

10 T. Носнмuтн, Forschungsber. L. Nordrhein-Westf. Nr. 1547 [1965].
2. von der Gleichheit der Verstärkungsfaktoren der beiden Differenzverstärker und der Verstärker im Oszillographen,

3. von der Ablenkgenauigkeit des Oszillographen,

4. von der Konstanz des Entladungsstroms $I_{\mathrm{a}}$.

Addiert man die Fehler, so erhält man in der stabilen positiven Säule ohne Streifen einen Fehler der absoluten Potentialdifferenzen $<5 \%$. Voraussetzung dafür ist eine genaue Eichung der Spannungsteiler und vor allem eine fortlaufende Nullpunktskontrolle der Verstärker während der Messung.

\section{b) Messung der elektrischen Feldstärke- und Strom- Schwankungen}

Für eine genaue Feststellung des Einsatzpunktes der K-Instabilität ist es nötig, die Wechselspannungsamplituden zwischen jeweils 2 Sonden direkt zu registrieren. Dazu werden, wie aus Abb. 8 ersichtlich, die Wechselspannungsamplituden jedes Sondenkanals über einen variablen Kopplungskondensator und einen Amplituden- und Phasenabgleicher (A bzw. P) auf einen Eingang eines von zwei Differenzverstärkern $\mathrm{D}_{2}$ gegeben, deren Ausgangssignale ebenfalls oszillographiert werden. Die obere Übertragungsgrenze der Anordnung liegt unter Berücksichtigung des Sondeninnenwiderstandes bei $200 \mathrm{kHz}$. Die untere Grenzfrequenz ist durch Veränderung der Kopplungskondensatoren in 3 Stufen variabel $(2 \mathrm{kHz}, 100 \mathrm{~Hz}, 2 \mathrm{~Hz})$.
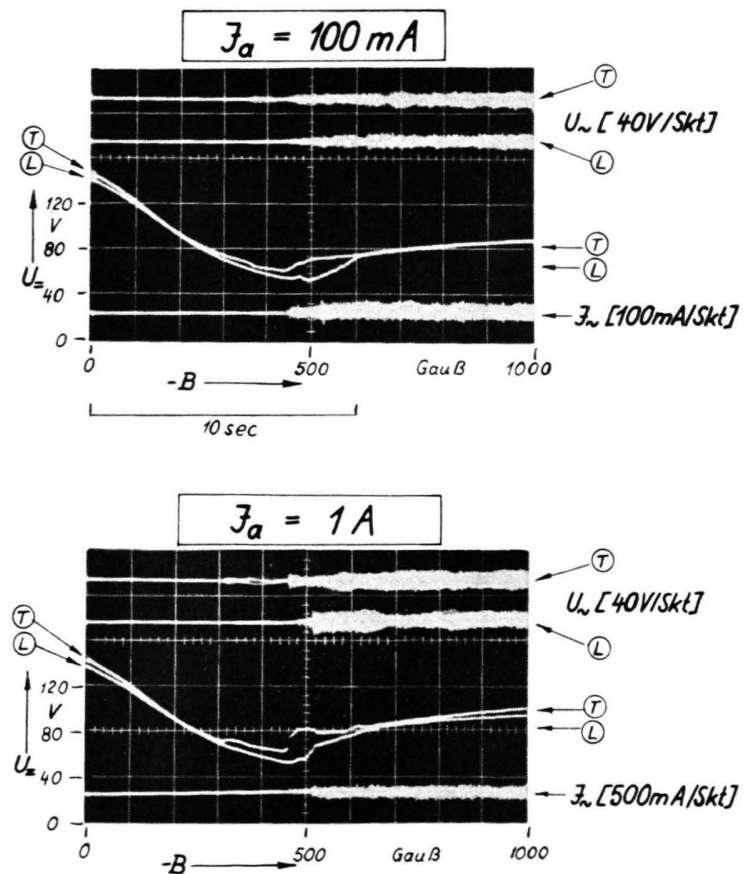

Abb. 9. Oszillogramme der longitudinalen elektrischen Feldstärken $E_{\|} \sim U_{\|}$und Rauschamplituden $U_{\sim}$ und $I_{\sim}$ in Abhängigkeit von der Magnetfeldstärke $B$ (Helium, $p=10^{-1}$ Torr).

11 W. L. Granowski, Elektrischer Strom im Gas, AkademieVerlag, Berlin 1955. 
Zur Registrierung eines ungefähren Maßes von schnellen Stromschwankungen wird mittels eines Zangenstromwandlers der Wechselstromanteil der Entladung abgenommen und über einen Verstärker $V_{2}$ auf einen weiteren Strahl des Oszillographen gegeben. An Hand der Höhe der Stromamplitude kann festgestellt werden, wann der gesamte Entladungskanal instabil ist. Ein Beispiel eines Oszillographenbildes zeigt Abb. 9.

\section{c) Messung der Elektronentemperatur und der Elektronendichte}

Für die Berechnung der Diffusionskoeffizienten ist die Kenntnis der Elektronentemperatur notwendig. Sie kann aus der Strom-Spannungs-Charakteristik einer ebenen Sonde entnommen werden. Die Langmurnsche Sondentheorie liefert hierzu die Beziehung

$$
I_{\mathrm{s}}=f_{\mathrm{s}} \cdot\left(I_{\mathrm{e}, \mathrm{st}} \cdot \exp \left(\frac{e\left(U_{\mathrm{s}}-U_{\mathrm{p}}\right)}{k T_{\mathrm{e}}}\right)+I_{\mathrm{i}}\right) \text { für } U_{\mathrm{s}}<U_{\mathrm{p}},
$$

wobei $I_{\mathrm{s}}=$ Sondenstrom, $f_{\mathrm{s}}=$ Sondenfläche, $I_{\mathrm{e}}$, st $=$ Elektronensättigungsstrom, $I_{\mathrm{i}}=$ Ionenstrom, $U_{\mathrm{s}}=$ Sondenpotential, $U_{\mathrm{p}}=$ Plasmapotential und $T_{\mathrm{e}}=$ Elektronentemperatur am Ort der Sonde (vgl. ${ }^{11,12}$ ).

In der Sondencharakteristik ist demnach die Steigung des Logarithmus des Elektronenstroms umgekehrt proportional zur Elektronentemperatur. Daraus ergibt sich folgende Schaltung zur Messung der Elektronentemperatur (Abb. 10). Die ebene „Löffel“-Sonde, deren Fläche $(2 \mathrm{~mm} \phi)$ in der Mitte des Krümmers auf der Achse der Entladung zur Anode hin orientiert ist, wird

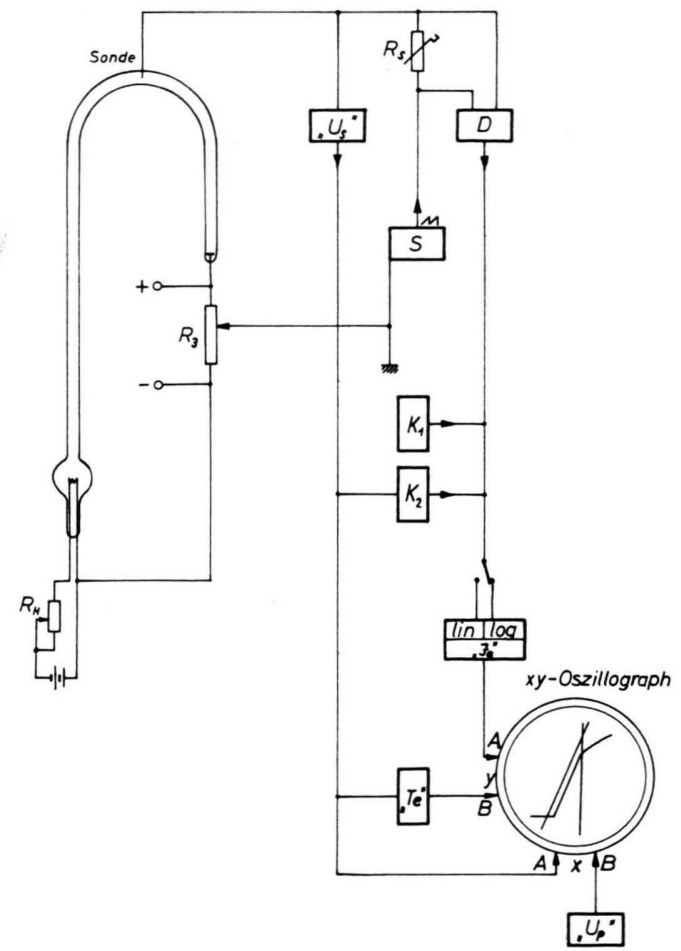

Abb. 10. Messung von Elektronentemperatur und -dichte.

12 R. S. Harp, Rev. Sci. Instr. 34, 416 [1963]. von einem Sägezahngenerator S (Amplitude: $120 \mathrm{~V}$, Frequenz: 0,1 bis $1000 \mathrm{~Hz}$ ) über einen Meßwiderstand $\mathrm{R}_{\mathrm{S}}$ gespeist. Über einen Impedanzwandler , $U_{\mathrm{S}}$ “ gelangt die Sondenspannung $1: 1$ auf einen $x$-Eingang (A) eines $x y$-Oszillographen. Ein dem Sondenstrom proportionales Signal wird an $R_{S}$ abgegriffen und an die beiden Eingänge eines Differenzverstärkers D geführt. Zur Kompensation des Ionenstroms wird zu dem Ausgangssignal von $\mathrm{D}$ eine konstante Spannung von $\mathrm{K}_{1}$ und eine zu $U_{\mathrm{s}}$ proportionale Spannung von $\mathrm{K}_{2}$ addiert. Über den logarithmischen Teil des Verstärkers „I “" wird das Signal schließlich auf einen $y$-Eingang (A) des Oszillographen gegeben. Ein Teil der Ablenkspannung gelangt über einen kontinuierlich variablen, von außen kontrollierbaren Abschwächer „ $T_{\mathrm{e}}$ “ auf einen zweiten $y$-Eingang (B) des Oszillographen. Durch Paralleljustierung dieses Strahls zum geraden Teil der logarithmischen Elektronenstromcharakteristik läßt sich die Elektronentemperatur am geeichten Einstellpotentiometer von "T $T_{\mathrm{e}}$ " ablesen (Abb. 11). Dadurch kann der (elektronische) Fehler der Elektronentemperaturmessung auf etwa $5 \%$ gedrückt werden.

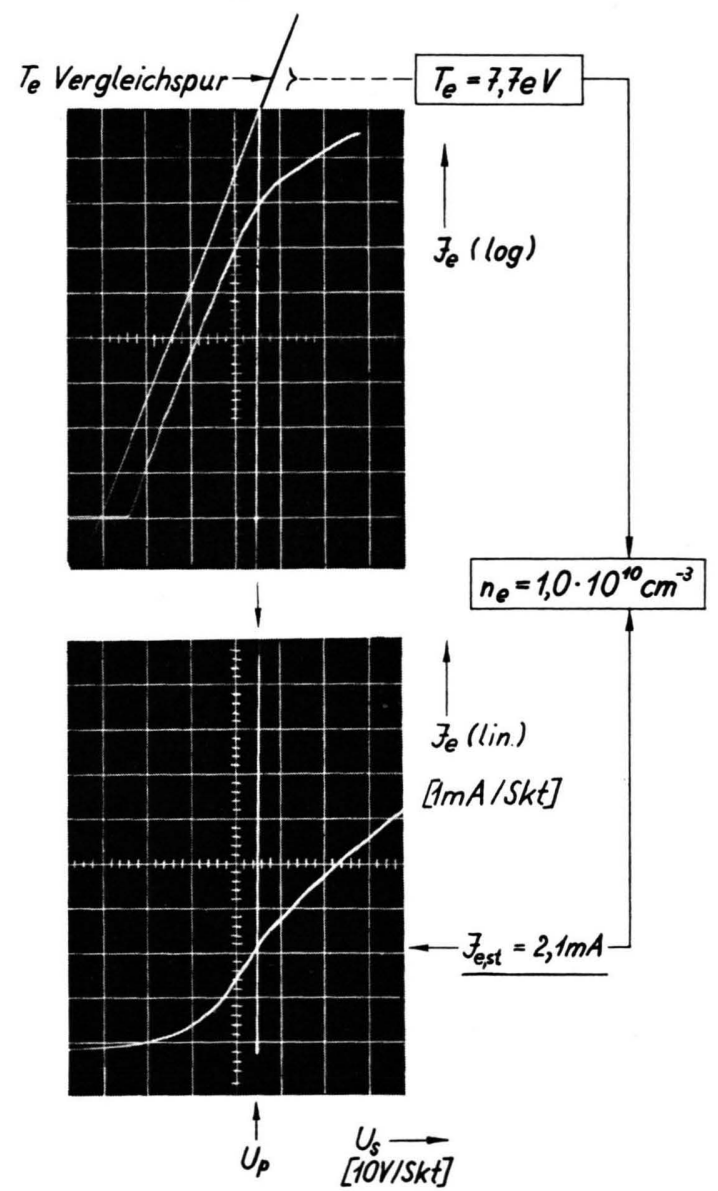

Abb. 11. Oszillogramme für die Bestimmung von Elektronentemperatur und -dichte (Helium, $p=3 \cdot 10^{-1}$ Torr, $I_{\mathrm{a}}=1,5 \mathrm{~A}$, $B=0)$. 
Zur Messung der Elektronendichte wird Gebrauch gemacht von der bekannten Beziehung

$$
L_{\mathrm{e}, \mathrm{st}}=n_{\mathrm{e}} f_{\mathrm{s}} e \sqrt{k T_{\mathrm{e}} / 2 \pi m_{\mathrm{e}}},
$$

wobei $n_{\mathrm{e}}=$ Elektronendichte am Ort der Sonde, $m_{\mathrm{e}}=$ Elektronenmasse.

Der Elektronensättigungsstrom $I_{\mathrm{e}}$, st wird bestimmt durch Markierung des Knicks der logarithmischen Kennlinie mit Hilfe einer konstanten Spannung von " $U_{\mathrm{p}}$ ", die auf einen weiteren $x$-Eingang (B) des Oszillographen gegeben wird, und durch darauf folgendes Umschalten von " $I_{\mathrm{e}}$ “ auf linearen Betrieb. Der Wert von $I_{\mathrm{e}}$, st kann dann bequem an der Stelle der „U, $U_{\mathrm{p}}$ "-Marke abgelesen werden (vgl. Abb. 11). $n_{\mathrm{e}}$ wird anschließend unter Benützung des gemessenen $T_{\mathrm{e}}$-Wertes berechnet. Die durch die Elektronik und Ablesung bedingte Ungenauigkeit der $n_{\mathrm{e}}$-Werte liegt unter $10 \%$.

Damit die Genauigkeit bei der Aufnahme der Sondenkennlinie nicht beinträchtigt wird, muß die störende 4 V-Wechselspannung des Heiztransformators durch Verwendung eines Heiz-Akkumulators beseitigt werden. Außerdem wird vor jeder Messung das Sondenpotential mit Hilfe von $R_{3}$ auf Erdpotential gebracht. Es muß $R_{3}$ verwendet werden, da der zu geringe Querstrom von $R_{4}$ bei einem Sondenstrom bis zu einigen $m A$ eine Verfälschung der Sondenkennlinie zur Folge hätte. Da die Magnetfeldstärke bei $T_{\mathrm{e}^{-}}$und $n_{\mathrm{e}}$-Messungen konstant bleibt, hat die Verwendung von $\mathrm{R}_{3}$ keinen Einfluß auf die Konstanz von $I_{\mathrm{a}}$.

\section{d) Spektroskopische Messungen}

Messungen mit einem Handspektroskop wurden dazu benutzt, um die Reinheit des Plasmas zu überprüfen und den Farbumschlag in der positiven Säule beim Einsetzen der K-Instabilität bei niedrigen Drucken zu untersuchen. Das Handspektroskop konnte praktisch an allen interessierenden Punkten der Entladung senkrecht zur Achse angesetzt werden, da die Schlitze in den Magnetfeldspulen (2 mm Breite im zylindrischen Teil, $4 \mathrm{~mm}$ im toroidalen Teil) eine Durchsicht ermöglichten.

In unmittelbarer Nähe der Kathode wurden Spuren von Metalldämpfen festgestellt, wenn die Kathode durch langen Betrieb inaktiviert war und zur Erreichung des Sättigungsstromes auf hohe Temperaturen geheizt werden mußte. In diesem Falle wurde die Kathode gegen eine neue ausgetauscht.

\section{Ergebnisse}

\section{Theoretische Beschreibung}

a) Zylindersymmetrische positive Säule

Für die Berechnung der Ladungsträgerverluste im zylindrischen Teil von AMBIPOL gehen wir nach bekannter Methode ${ }^{13}$ aus von den Gleichungen für

13 A. Schlëter, Z. Naturforschg. 6 a, 73 [1951]. die Impuls-, Ladungs- und Massenbilanz für ein Dreikomponentenplasma bestehend aus Neutralgasatomen, einfach geladenen positiven Ionen der gleichen Sorte und Elektronen ${ }^{14}$. Dabei machen wir die bei der Behandlung der positiven Säule einer Glimmentladung üblichen Annahmen ${ }^{13,14}$ (u. a.: Quasineutralität; Vernachlässigung der inneren Reibung der einzelnen Komponenten, von Mehrfachstößen, metastabilen Zuständen, Thermodiffusion, Strahlungsdruck, Gravitationswirkung und - wegen des schwachen Ionisationsgrades - auch Vernachlässigung der Coulomв-Stöße und der Volumenrekombination). Die makroskopische Bewegung der Plasmakomponenten sei so langsam, daß alle Relaxationen nur durch Diffusion bestimmt sind und Trägheitsglieder nicht berücksichtigt zu werden brauchen. Unter Benützung dieser Näherungen und mit der Annahme, daß sich das Neutralgas makroskopisch in Ruhe befindet, erhalten wir als Bewegungsgleichungen für einfach geladene Ionen:

$$
\begin{array}{r}
e n\left(\boldsymbol{E}+\boldsymbol{v}_{\mathrm{i}} \times \boldsymbol{B}\right)-\nabla\left(k T_{i} n\right)-v_{\mathrm{i}} m_{\mathrm{i}} n \boldsymbol{v}_{\mathrm{i}}=0, \\
-e n\left(\boldsymbol{E}+\boldsymbol{v}_{\mathrm{e}} \times \boldsymbol{B}\right)-\nabla\left(k T_{\mathrm{e}} n\right)-v_{\mathrm{e}} m_{\mathrm{e}} n \boldsymbol{v}_{\mathrm{e}}=0,
\end{array}
$$

wobei $\boldsymbol{v}_{\mathrm{i}}, \boldsymbol{v}_{\mathrm{e}}$ die makroskopischen Geschwindigkeiten der Ionen bzw. Elektronen, $T_{\mathrm{i}}, T_{\mathrm{e}}$ die Temperaturen des Ionen- bzw. Elektronen-Gases (die als ideale Gase beschreibbar angenommen werden) und $n=n_{\text {i, e }}$ die Dichte des (quasineutralen) Plasmas sind; die Stoßfrequenzen zwischen Ion bzw. Elektron und Neutralgasatomen werden durch $v_{\mathrm{i}}$ bzw. $v_{\mathrm{e}}$ dargestellt, die unabhängig von den Geschwindigkeiten sein sollen; die übrigen Symbole haben ihre konventionelle Bedeutung.

Die Vernachlässigung der Neutralgasgeschwindigkeit $\boldsymbol{v}_{\mathrm{n}}$ ist möglich, da in AMBIPOL $v_{\mathrm{n}}=15 / p$ $[\mathrm{cm} / \mathrm{s}]$ ( $p$ in Torr) klein gegen die anderen interessierenden Geschwindigkeiten ist.

Bei Einzel-Stoß-Erzeugung von einfach geladenen Ionen mit der Rate $\xi$ pro Elektron lauten die Kontinuitätsgleichungen:

$$
\xi \cdot n-\frac{\partial n}{\partial t}=\nabla\left(n \boldsymbol{v}_{\mathrm{i}}\right)=\nabla\left(n \boldsymbol{v}_{\mathrm{e}}\right) .
$$

Die Gln. (1) und (2) liefern für die zum Magnetfeld parallelen ( $\|$ ) Geschwindigkeitskomponenten:

$$
\begin{aligned}
& \boldsymbol{v}_{\mathrm{i} \|}=b_{\mathrm{i}} \boldsymbol{E}_{\|}-(1 / n) \nabla_{\|}\left(D_{\mathrm{i}} n\right), \\
& \boldsymbol{v}_{\mathrm{e} \|}=-b_{\mathrm{e}} \boldsymbol{E}_{\|}-(1 / n) \nabla_{\|}\left(D_{\mathrm{e}} n\right),
\end{aligned}
$$

14 B. Lehnert, Proc. 2nd Intern. Conf. Peaceful Uses Atom. Energy UN, New York 32, 349 [1958]. 
wobei

$$
b_{\mathrm{i}}=e / m_{\mathrm{i}} v_{\mathrm{i}} \quad \text { und } \quad b_{\mathrm{e}}=e / m_{\mathrm{e}} \boldsymbol{v}_{\mathrm{e}}
$$

die Werte der Beweglichkeiten und

$$
D_{\mathrm{i}}=k T_{\mathrm{i}} / m_{\mathrm{i}} v_{\mathrm{i}} \quad \text { und } \quad D_{\mathrm{e}}=k T_{\mathrm{e}} / m_{\mathrm{e}} v_{\mathrm{e}}
$$

die Diffusionskoeffizienten für Ionen bzw. Elektronen sind.

Für die zu $\boldsymbol{B}$ senkrechten $(\perp)$ Komponenten erhält man nach vektorieller Multiplikation von Gl. (1)

und (2) mit $\boldsymbol{B}$ und Kombination mit (4) und (5) :

$$
\begin{aligned}
& \left(1+\frac{1}{y_{\mathrm{i}}^{2}}\right) \boldsymbol{v}_{\mathrm{i} \perp}=\frac{\boldsymbol{E} \times \boldsymbol{B}}{B^{2}}+\frac{1}{y_{\mathrm{i}} n} \frac{\boldsymbol{B}}{B} \times \nabla\left(D_{\mathrm{i}} n\right)-\frac{1}{y_{\mathrm{i}}{ }^{2} n} \nabla_{\perp}\left(D_{\mathrm{i}} n\right)+\frac{1}{y_{\mathrm{i}}{ }^{2}} b_{\mathrm{i}} \boldsymbol{E}_{\perp}, \\
& \left(1+\frac{1}{y_{\mathrm{e}}{ }^{2}}\right) \boldsymbol{v}_{\mathrm{e} \perp}=\frac{\boldsymbol{E} \times \boldsymbol{B}}{B^{2}}-\frac{1}{y_{\mathrm{e}} n} \frac{\boldsymbol{B}}{B} \times \nabla\left(D_{\mathrm{e}} n\right)-\frac{1}{y_{\mathrm{e}}{ }^{2} n} \nabla_{\perp}\left(D_{\mathrm{e}} n\right)-\frac{1}{y_{\mathrm{e}}{ }^{2}} b_{\mathrm{e}} \boldsymbol{E}_{\perp},
\end{aligned}
$$

wobei $y_{\mathrm{i}}=\omega_{\mathrm{i}} / \nu_{\mathrm{i}}$ und $y_{\mathrm{e}}=\omega_{\mathrm{e}} / \nu_{\mathrm{e}}$ mit $\omega_{\mathrm{i}}=e B / m_{\mathrm{i}}$ und $\omega_{\mathrm{e}}=e B / m_{\mathrm{e}}$, den Gyrationsfrequenzen von Ionen bzw. Elektronen.

Beim Einsetzen von (6) und (7) in (3) machen wir von der Bedingung der Stationarität der Entladung Gebrauch, indem wir das elektrische Feld als Gradient eines elektrischen Potentials $(\boldsymbol{E}=-\nabla V)$ schreiben und partielle Zeitableitungen weglassen. Außerdem werden wir das Eigenmagnetfeld der Entladung vernachlässigen, indem wir das Magnetfeld homogen und parallel zur Entladungsachse annehmen, und nur den radialen Teilchenfluß berücksichtigen, da die axialen Abmessungen der Entladung groß gegen ihre radialen sind. Gl. (3) nimmt dann die Gestalt an:

$$
\xi n=\nabla\left(n \boldsymbol{v}_{\mathrm{i} \perp}\right)=\nabla\left(n \boldsymbol{v}_{\mathrm{e} \perp}\right) ;
$$

bei der Divergenzoperation (8) an (6) und (7) fallen jeweils die beiden ersten Glieder auf der rechten Seite weg, da $\nabla V \times \nabla n$ bei axialer Symmetrie keine Komponente in B-Richtung enthält, und wir erhalten bei Annahme von über den Radius konstanten Temperaturen $T_{\mathrm{i}}$ und $T_{\mathrm{e}}$ :

$$
\begin{aligned}
& \left(1+\frac{1}{y_{\mathrm{i}}{ }^{2}}\right) \nabla \cdot\left(n \boldsymbol{v}_{\mathrm{i} \perp}\right)=-\frac{1}{y_{\mathrm{i}}{ }^{2}} D_{\mathrm{i}} \nabla \cdot(\nabla \perp n)-\frac{1}{y_{\mathrm{i}}{ }^{2}} b_{\mathrm{i}} \nabla \cdot\left(n \nabla_{\perp} V\right), \\
& \left(1+\frac{1}{y_{\mathrm{e}}{ }^{2}}\right) \nabla \cdot\left(n \boldsymbol{v}_{\mathrm{e} \perp}\right)=-\frac{1}{y_{\mathrm{e}}{ }^{2}} D_{\mathrm{e}} \nabla \cdot(\nabla \perp n)+\frac{1}{y_{\mathrm{e}}{ }^{2}} b_{\mathrm{e}} \nabla \cdot\left(n \nabla_{\perp} V\right) .
\end{aligned}
$$

Die Kombination von (8), (9) und (10) liefert nach Elimination von $V$ :

$$
\xi_{\mathrm{L}} n=-D_{\mathrm{a} \perp} \nabla_{\perp}^{2} n,
$$

wobei

$$
\begin{aligned}
D_{\mathrm{a} \perp} & =\frac{k\left(T_{\mathrm{i}}+T_{\mathrm{e}}\right)}{m_{\mathrm{i}} \nu_{\mathrm{i}}\left(1+y_{\mathrm{i}}{ }^{2}\right)+m_{\mathrm{e}} \nu_{\mathrm{e}}\left(1+y_{\mathrm{e}}{ }^{2}\right)} \\
& =\frac{k\left(T_{\mathrm{i}}+T_{\mathrm{e}}\right) b_{\mathrm{i}} / e}{1+y_{\mathrm{i}}^{2}+\left(b_{\mathrm{i}} / b_{\mathrm{e}}\right)\left(1+y_{\mathrm{e}}{ }^{2}\right)} \\
& \approx \frac{k T_{\mathrm{e}} b_{\mathrm{i}} / e}{1+\left(b_{\mathrm{i}} / b_{\mathrm{e}}\right) y_{\mathrm{e}}{ }^{2}}=\frac{k T_{\mathrm{e}} b_{\mathrm{i}} / e}{1+b_{\mathrm{i}} b_{\mathrm{e}} B^{2}}
\end{aligned}
$$

$$
\text { (wegen } T_{\mathrm{i}} \ll T_{\mathrm{e}}, b_{\mathrm{i}} \ll b_{\mathrm{e}} \text { ) }
$$

der transversale ambipole Diffusionskoeffizient ist. (Der Index $\mathbf{L}$ bezeichnet die Ionisationsrate im linearen Teil der Entladung.)

Gl. (11) beschreibt das Gleichgewicht zwischen Ladungsträgerproduktion (linke Seite) und Ladungsträgerverlust (rechte Seite) durch die mit gleicher
Geschwindigkeit zur Wand diffundierenden Elektronen und Ionen (ambipolare Diffusion). Für eine zylindrische von nichtleitenden $\mathrm{W}$ änden eingeschlossene Plasmasäule (Abb. 12, System L), deren Radius $a$ groß gegen die freien Weglängen der Elektronen $\lambda_{\mathrm{e}}$ und Ionen $\lambda_{\mathrm{i}}$ ist (was für AMBIPOL erfüllt ist), lautet die physikalisch realisierbare auf der Achse reguläre Lösung von (11), die die Randbedingung $n(r=a)=0$ erfüllt:

$$
n(r)=n_{0} \cdot J_{0}\left(r / A_{0}\right)
$$

mit

$$
\Lambda_{0}=a / \gamma_{0}=\sqrt{\left.D_{\mathrm{i} \mathrm{L}}\right\lrcorner / \xi_{\mathrm{L}}}, \quad \gamma_{0}=2,504,
$$

wobei $n_{0}=n(r=0)$ die Dichte auf der Achse, $J_{0}$ die Bessed-Funktion nullter Ordnung und $\gamma_{0}$ deren erste Nullstelle sind.

Die für die Ladungsträgerverluste repräsentative Ionisationsrate $\xi_{\mathrm{L}}=D_{\mathrm{a} \perp} / \Lambda_{\mathbf{0}}^{2}$ fällt demnach für hohe Magnetfeldstärken mit $1 / B^{2}$ ab. Die tatsächliche 
Höhe dieser Verluste ist wegen der gleichzeitigen Erniedrigung von $T_{\mathrm{e}}$ und Erhöhung von $n$ mit wachsendem $B$ nur über eine Energiebilanz der positiven Säule zu errechnen $\left(\right.$ siehe $\left.{ }^{14}\right)$.

\section{b) Vollionisiertes Plasma im toroidalen Magnetfeld}

Die in Abb. 3 gezeigte Torusdrift kann im mikroskopischen Bild für $n k T /\left(B^{2} / 8 \pi\right)=, \beta^{\prime \prime} \ll 1$ leicht berechnet werden ${ }^{15}$. Wir verwenden dabei die in Abb. 12, System T, gezeigte Geometrie. Das Magnetfeld $\boldsymbol{B}$ weise in Richtung $\vartheta$ und habe die Stärke

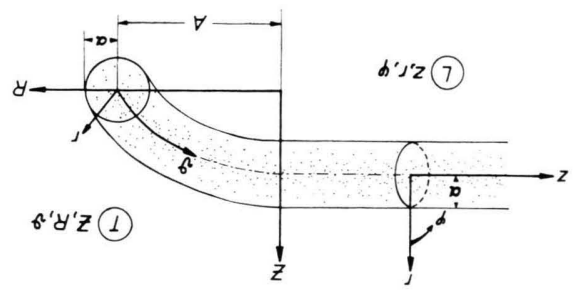

Abb. 12. Koordinatensystem für den linearen bzw. den toroidalen Teil.

$B_{0} \cdot A / R$. Wenn wir für die „magnetische DipolKraft" auf ein gyrierendes Teilchen das Produkt

$$
\text { magn. Moment } \cdot\left|\nabla_{\perp} B\right|=\frac{1}{2} m_{\mathrm{q}} u_{\mathrm{q} \perp}^{2} / R
$$

setzen $\left(u_{\mathrm{q}} \perp=\right.$ thermische Geschwindigkeit des Teilchens senkrecht zu B) und die Zentrifugalkraft mit $m_{\mathrm{q}} u_{\mathrm{q} \|}^{2} / R$ berücksichtigen ${ }^{15}$, erhalten wir aus der Kräftebilanz für das Gyrationszentrum:

$$
\frac{1}{2} \frac{m_{\mathrm{q}} u_{\mathrm{q}}^{2} \perp}{R}+\frac{m_{\mathrm{q}} u_{\mathrm{q} \|}^{2} \|}{R}= \pm m_{\mathrm{q}} \omega_{\mathrm{q}} v_{\mathrm{q} z}+m_{\mathrm{q}} \frac{\mathrm{d} v_{\mathrm{q} R}}{\mathrm{~d} t},
$$

wobei der Index q für $\mathrm{i}(+)$ und $\mathrm{e}(-)$ steht und $\mathrm{d} v_{q R} / \mathrm{d} t$ die Beschleunigung des Führungszentrums für einen mitbewegten Beobachter in Richtung $R$ darstellt $\left(\boldsymbol{v}_{\mathrm{q} R}=\boldsymbol{v}_{\mathrm{i} R}=\boldsymbol{v}_{\mathrm{e} R}=\left[\begin{array}{l}\boldsymbol{E} \times \boldsymbol{B}\end{array}\right] / B^{2}(\mathrm{Abb} .3)\right)$.

Im Falle der Gleichverteilung der kinetischen Energie können wir $m_{\mathrm{q}}\left(\frac{1}{2} u_{\mathrm{q} \perp}^{2}+u_{\mathrm{q} \|}^{2}\right)=2 k T_{\mathrm{q}}$ schreiben und erhalten mit $\mathrm{d} v_{\mathrm{q} R} / \mathrm{d} t=\alpha_{R}$ :

$$
\begin{aligned}
& v_{\mathrm{i} Z}=(1 / e B)\left(2 k T_{\mathrm{i}} / R-m_{\mathrm{i}} \alpha_{R}\right), \\
& v_{\mathrm{e} Z}=(1 / e B)\left(-2 k T_{\mathrm{e}} / R+m_{\mathrm{e}} \alpha_{R}\right) .
\end{aligned}
$$

15 P. C. T. van der LaAn, Plasma Phys. (J. Nucl. Energy, Part C) 6, 559 [1964].
Mit der Forderung, daß die makroskopische Stromdichte in $Z$-Richtung $j_{Z}=n e\left(v_{\mathrm{i} Z}-v_{\mathrm{e} Z}\right)$ verschwinden soll (nichtleitende Wände!), ergibt sich durch Subtraktion der Gl. (17) von Gl. (18)

$$
\alpha_{R}=2 k\left(T_{\mathrm{i}}+T_{\mathrm{e}}\right) /\left[R\left(m_{\mathrm{i}}+m_{\mathrm{e}}\right)\right]
$$

als Beschleunigung des Plasmas in R-Richtung.

\section{c) Torusdrift im schwach ionisierten Plasma}

Für den Fall des schwach ionisierten Plasmas wird die beschleunigte Bewegung $\alpha_{R}$ des Plasmas durch die "Reibung“ am Neutralgas auf eine konstante Geschwindigkeit $w_{\mathrm{q} R}$ abgebremst werden. Wir können diese Geschwindigkeit für $\omega_{\mathrm{i}} \omega_{\mathrm{e}} \gg v_{\mathrm{i}} v_{\mathrm{e}}$ und $w_{\mathrm{q} R} \ll \sqrt{T_{\mathrm{q}} / m_{\mathrm{q}}}$ näherungsweise berechnen, wenn wir die Bilanz der Summen der beschleunigenden und der Reibungskräfte ansetzen:

$$
\left(m_{\mathrm{i}}+m_{\mathrm{e}}\right) \alpha_{R}=v_{\mathrm{i}} m_{\mathrm{i}} w_{\mathrm{i} R}+v_{\mathrm{e}} m_{\mathrm{e}} w_{\mathrm{e} R} .
$$

Da die resultierende zeitunabhängige Drift ambipolar ist $\left(w_{\mathrm{i} R}=w_{\mathrm{e} R}=w_{R}\right)$, läßt sie sich schreiben als

$$
\begin{aligned}
w_{R}=\frac{\left(m_{\mathrm{i}}+m_{\mathrm{e}}\right) \alpha_{R}}{v_{\mathrm{i}} m_{\mathrm{i}}+v_{\mathrm{e}} m_{\mathrm{e}}}=\frac{2 k\left(T_{\mathrm{i}}+T_{\mathrm{e}}\right)}{R\left(v_{\mathrm{i}} m_{\mathrm{i}}+v_{\mathrm{e}} m_{\mathrm{e}}\right)} \\
=\frac{2 D_{\mathrm{a} \perp}(B=0)}{R} \approx \frac{2 k T_{\mathrm{e}} b_{\mathrm{i}} / e}{A}
\end{aligned}
$$

für

$$
T_{\mathrm{i}} \ll T_{\mathrm{e}}, b_{\mathrm{i}} \ll b_{\mathrm{e}}, v_{\mathrm{i}} \cdot v_{\mathrm{e}} \ll \omega_{\mathrm{i}} \cdot \omega_{\mathrm{e}}, a \ll A .
$$

Dieser Wert muß nun bei der Divergenzbildung in Gl. (8) noch berücksichtigt werden. Da die transversalen Abmessungen des toroidalen Entladungsgefäßes klein gegen seine longitudinalen sind, erhält man die der Gl. (11) entsprechende lineare homogene Differentialgleichung vom elliptischen Typ:

$$
\xi_{\mathbf{T}} n=\nabla\left(n \boldsymbol{v}_{\mathrm{e}}\right)=-D_{\mathrm{a} \perp} \nabla_{\perp}^{2} n+w_{R} \frac{\partial n}{\partial R}
$$

oder in Zylinderkoordinaten:

$D_{\mathrm{a} \perp}\left(\frac{\partial^{2} n}{\partial R^{2}}+\frac{\partial^{2} n}{\partial Z^{2}}\right)-\left(w_{R}-\frac{D_{\mathrm{a}} \perp}{R}\right) \frac{\partial n}{\partial R}+\xi_{\mathrm{\tau}} n=0$,

wobei die Invarianz des Problems bezgl. Drehungen um die $Z$-Achse vorausgesetzt wird.

Für die Lösung dieser Gleichung, die die Randbedingung $n(r=a)=0$ erfüllt, machen wir folgenden Ansatz:

$$
n=\sum_{\mu} c_{\mu} f_{\mu}(R, Z) \exp \left(\eta_{\mu} R\right),
$$


wobei die $f_{\mu}$ die Eigenfunktionen der Gleichung

$$
\nabla_{\perp}^{2} f+\left(1 / \Lambda^{\prime 2}\right) f=0
$$

sind, mit $1 / \Lambda_{\mu}{ }^{\prime 2}$ als den zugehörigen Eigenwerten, die durch die obige Randbedingung bestimmt sind.

Setzen wir (25) und (26) in (24) ein und berücksichtigen $D_{\mathrm{a} \perp} / R \ll w_{R}$ wegen $\omega_{\mathrm{i}} \omega_{\mathrm{e}} \gg v_{\mathrm{i}} v_{\mathrm{e}}(22)$, so erhalten wir:

$$
\sum_{\mu} c_{\mu}\left(\left(D_{\mathrm{a} \perp} / \Lambda_{\mu}^{\prime 2}-D_{\mathrm{a} \perp} \eta_{\mu}^{2}+w_{R} \eta_{\mu}-\xi_{\mathrm{T}}\right) f_{\mu}+\left(-2 D_{\mathrm{a} \perp} \eta_{\mu}+w_{R}\right) \partial f_{\mu} / \partial R\right)=0 .
$$

Für eine spezielle Lösung berücksichtigen wir nur das erste Glied der Summe. Dann verschwindet wegen (26) $\partial f_{0} / \partial R$ für $r=a$ im allgemeinen nicht. Auf Grund der Randbedingungen ist jedoch $f_{0}(r=a)=0$, d. h. $-2 D_{\mathrm{a} \perp} \eta_{0}+w_{R}=0$ für $r=a$ und, da in erster Näherung $(A \gg a)$ die Größen $w_{R}$ und $D_{\mathrm{a} \perp}$ unabhängig von $r$ sind,

$$
\eta_{0} \approx w_{R} / 2 D_{\mathrm{a} \perp} \text { für alle } r \leqq a .
$$

Wegen Gl. (26) ist i. allg. $f_{0}(r<a) \neq 0$, d. h. in (27) ist

$$
D_{\mathrm{a} \perp} / \Lambda_{0}^{\prime 2}-D_{\mathrm{a} \perp} \eta_{0}^{2}+w_{R} \eta_{0}-\xi_{\mathrm{T}}=0 \quad \text { für alle } \quad r<a .
$$

Setzen wir (28) in (29) ein und schreiben wegen $A \gg a$ nach (11) und (14) für $\Lambda_{0}^{\prime} \approx A_{0}=a / \gamma_{0}$, erhalten wir:

$$
\xi_{\mathbf{T}}=\frac{D_{\mathrm{a} \perp}}{a^{2} / \gamma_{0}^{2}}+\frac{w_{R}^{2}}{4 D_{\mathrm{a} \perp}} \approx k T_{\mathrm{e}} \frac{b_{\mathrm{i}}}{e}\left(\frac{\gamma_{0}^{2}}{a^{2}} \frac{1}{\left(b_{\mathrm{i}} / b_{\mathrm{e}}\right) y_{\mathrm{e}}^{2}}+\frac{1}{A^{2}} \frac{b_{\mathrm{i}}}{b_{\mathrm{e}}} y_{\mathrm{e}}^{2}\right),
$$

wenn $T_{\mathrm{i}} \ll T_{\mathrm{e}}, \quad b_{\mathrm{i}} \ll b_{\mathrm{e}}, \quad v_{\mathrm{i}} v_{\mathrm{e}} \ll \omega_{\mathrm{i}} \omega_{\mathrm{e}}, \quad$ d. h. $\quad\left(b_{\mathrm{i}} / b_{\mathrm{e}}\right) y_{\mathrm{e}}^{2} \gg 1$.

Für $B \rightarrow 0$, also $v_{\mathrm{i}} v_{\mathrm{e}} \gg \omega_{\mathrm{i}} \omega_{\mathrm{e}}$, geht dieser Ausdruck in

$$
k T_{\mathrm{e}} \frac{b_{\mathrm{i}}}{e}\left(\frac{\gamma_{0}^{2}}{a^{2}} \frac{1}{1+\left(b_{\mathrm{i}} / b_{\mathrm{e}}\right) y_{\mathrm{e}}^{2}}\right)
$$

über. Wir können demnach, um auch das Zwischengebiet $v_{\mathrm{i}} \nu_{\mathrm{e}} \approx \omega_{\mathrm{i}} \omega_{\mathrm{e}}$ mit einzuschließen, näherungsweise schreiben:

$$
\xi_{\mathbf{T}} \approx k T_{\mathrm{e}} \frac{b_{\mathrm{i}}}{e}\left(\frac{\gamma_{0}^{2}}{a^{2}} \frac{1}{1+\left(b_{\mathrm{i}} / b_{\mathrm{e}}\right) y_{\mathrm{e}}^{2}}+\frac{1}{A^{2}} \frac{b_{\mathrm{i}}}{b_{\mathrm{e}}} y_{\mathrm{e}}^{2}\right)=k T_{\mathrm{e}} \frac{b_{\mathrm{i}}}{e} \frac{\gamma_{0}^{2}}{a^{2}}\left(\frac{1}{1+b_{\mathrm{i}} b_{\mathrm{e}} B^{2}}+\frac{1}{\gamma_{0}^{2}} \frac{a^{2}}{A^{2}} b_{\mathrm{i}} b_{\mathrm{e}} B^{2}\right) .
$$

Die Ionisationsrate setzt sich also im toroidalen Fall zusammen aus dem linearen Anteil $\xi_{\mathbf{L}}$, den wir schon von Gl. (11) kennen, und einen mit $B^{2}$ ansteigenden Term, der die Verluste durch die Torusdrift repräsentiert. Für die relative Ionisationsrate im toroidalen Teil bezogen auf die im zylindrischen Teil ergibt sich

$$
\begin{aligned}
\Phi & =\xi_{\mathbf{T}} / \xi_{\mathbf{L}} \\
& \approx\left(T_{\mathrm{e}} \mathbf{T} / T_{\mathrm{e}} \mathbf{L}\right)\left(1+\frac{1}{\gamma_{0}^{2}} \frac{a^{2}}{A^{2}}\left(1+b_{\mathrm{i}} b_{\mathrm{e}} B^{2}\right) b_{\mathrm{i}} b_{\mathrm{e}} B^{2}\right)
\end{aligned}
$$

Das relative longitudinale elektrische Feld im toroidalen Teil bezogen auf das im geraden Teil $\Theta_{\text {theor }}(B)=\left[E_{\| \mathbf{T}}(B) / E_{\| \mathbf{L}}(B)\right]_{\text {theor }}$ läßt sich nur über eine Analyse der Energiebilanz von toroidalem und zylindrischem Teil der positiven Säule errech-

16 A. v. Engel u. M. Steenbeck, Elektrische Gasentladungen, Springer-Verlag, Berlin 1932, Bd. II, S. 86. nen. Wir können jedoch eine Abschätzung nach oben erhalten, wenn wir nach ${ }^{16}$ näherungsweise

$$
E_{\|} \sim T_{\mathrm{e}}{ }^{*}(p=\text { const }) \quad \text { mit } \quad 0<x<1
$$

setzen. Wegen der näherungsweise logarithmischen Abhängigkeit der Elektronentemperatur $T_{\mathrm{e}}$ von $\xi$ und wegen $\Phi>1$ ist dann

$$
\begin{aligned}
\Theta_{\text {theor }} & =\left(E_{\| \mathbf{T}} / E_{\| \mathbf{L}}\right)_{\text {theor }}<\Phi^{*}<\Phi \\
& \approx 1+\left(1 / \gamma_{0}^{2}\right)\left(a^{2} / A^{2}\right)\left(b_{\mathrm{i}} b_{\mathrm{e}} B^{2}+\left(b_{\mathrm{i}} b_{\mathrm{e}} B^{2}\right)^{2}\right)
\end{aligned}
$$

denn $T_{\mathrm{e}} / T_{\mathrm{e}} \approx 1$, solange $(\Phi-1)^{2} \ll 1$, d. h. für nicht zu große Magnetfeldstärken.

\section{Experimentelle Ergebnisse}

Nach der in B. III.c) beschriebenen Methode wurde die Elektronentemperatur in Abhängigkeit von der magnetischen und elektrischen Feldstärke bestimmt und mit (33) verglichen. Der Exponent 
von $T_{\mathrm{e}}$ ergab sich zu: $\varkappa=0,5$ bis 0,9 , wodurch die Abschätzung (34) legitimiert wird.

Da die Stromleitung in der positiven Säule fast nur von den Elektronen übernommen wird, kann durch die Messung der Plasmadichte $n_{0}$ auf der Entladungsachse die Beweglichkeit der Elektronen bestimmt werden. Für den Fall, daß das Dichteprofil nicht sehr von (13) abweicht, ist

$$
I_{\mathrm{a}}=2 \pi \cdot \int_{0}^{a} J_{0}\left(\gamma_{0} \cdot r / a\right) r \mathrm{~d} r \cdot n_{0} \cdot e b_{\mathrm{e}} E_{\|},
$$

woraus sich $b_{\mathrm{e}}=6,1 \cdot 10^{1 \tau} I_{\mathrm{a}} /\left(n_{0} E_{\|]}\right) \mathrm{cm} / \mathrm{Vs}$ ergibt. Die damit berechneten $b_{\mathrm{e}}$-Werte und die $b_{\mathrm{i}}$-Werte aus ${ }^{16}$ bzw. ${ }^{17}$ liegen Abb. 13 zugrunde, in der die

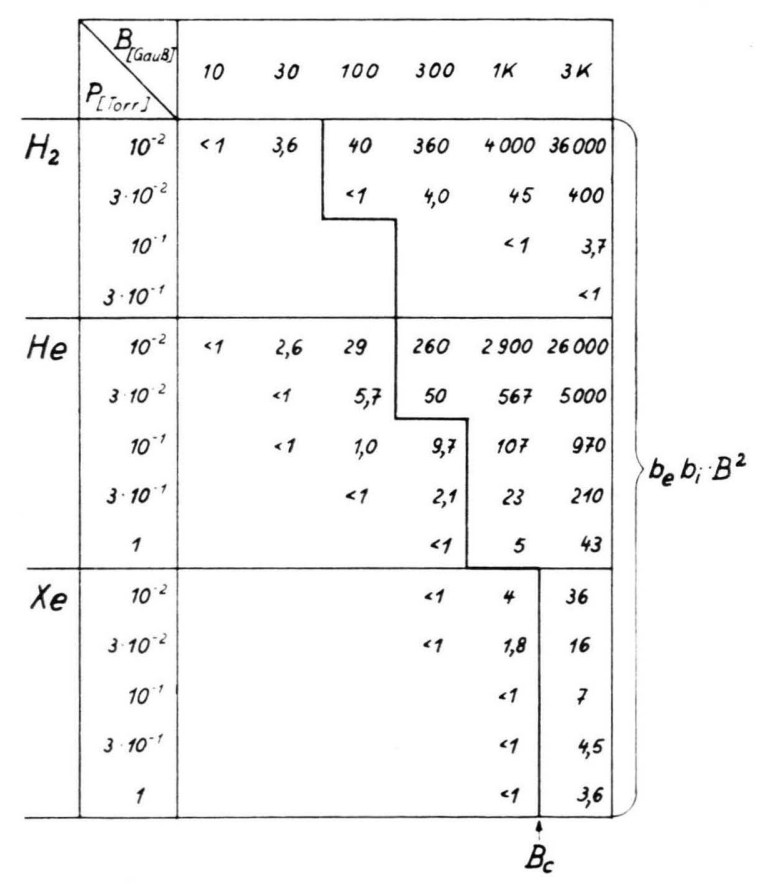

Abb. 13. Tabelle der Größe $b_{\mathrm{e}} b_{\mathrm{i}} B^{2}$ in Gl. (36).

für Gl. (34) bestimmende Größe $b_{\mathrm{i}} b_{\mathrm{e}} B^{2}=\omega_{\mathrm{i}} \omega_{\mathrm{e}} / v_{\mathrm{i}} v_{\mathrm{e}}$ tabelliert ist. Gl. (34) wird mit $\left[\left(1 / \gamma_{0}\right)(a / A)\right]^{2}$ $\approx 10^{-3} \mathrm{zu}:$

$$
\Theta_{\text {theor }}<\Phi \approx 1+10^{-3}\left(b_{\mathrm{i}} b_{\mathrm{e}} B^{2}+\left(b_{\mathrm{i}} b_{\mathrm{e}} B^{2}\right)^{2}\right) .
$$

In den Abbb. 14 bis 16 sind die Ergebnisse der nach der Methode B. III. a) und b) vorgenommenen Experimente gezeigt, wobei jeweils $U==E_{\|} \cdot 110 \mathrm{~cm}$ ist. Jedes Bild stellt den Mittelwert aus mindestens 2 Aufnahmen dar. Man sieht, daß $E_{\| \mathbf{L}}$ mit steigen-

17 S. C. Brows, Basic Data of Plasma Physics, Wiley \& Sons, New York 1959. dem $B$ in der von Lehnert ${ }^{14}$ vorausgesagten Form abfällt und weiter, daß $E_{\| \text {r }}$ für größere $B$ immer über $E_{\|\llcorner}$liegt, wie zu erwarten war. Für $B=0$ ist $E_{\| \mathbf{\iota}}$ im allgemeinen etwas höher als $E_{\| \mathbf{T}}$, was wahrscheinlich von dem radialen Gradienten von $E_{\|}$im toroidalen Teil der Entladung herrührt. Dieser würde - sofern diese Deutung zutrifft - zu einer kleinen Verschiebung des Dichteprofils zur inneren Wand des Entladungsgefäßes und dadurch zu einer Verminderung der effektiven Brennstrecke führen.

Bei Wasserstoff (Abb. 14) liegt der Einsatzpunkt der K-Instabilität (erkennbar am Beginn des Rauschens $U_{\sim}$ und am Minimum von $\left.U_{=}(B)\right]$ bei sehr niedrigen kritischen Magnetfeldstärken $B_{\mathrm{c}}$, für die die Theorie [Gl. (36) mit Tabelle Abb. 13] noch keinen merklichen Unterschied zwischen $E_{\| \mathbf{L}}$ und $E_{\| \boldsymbol{\top}}$ voraussagt. Für den turbulenten Zustand der Entladung oberhalb $B_{\mathrm{c}}$ ist die für die stabile positive Säule abgeleitete Torusdrift nicht mehr anwendbar. Außerdem werden die Messungen bei bestimmten Parametern der Wasserstoffentladung durch stehende Streifen gestört (vgl. Abb. 14, $I_{\mathrm{a}}=0,1 \mathrm{~A}, 3 \cdot 10^{-2}$ Torr und $10^{-1}$ Torr).

Die aus diesen Gründen vorgenommenen Experimente mit Helium (Abb. 15) liefern wegen des allgemein höheren $B_{\mathrm{c}}$ einen größeren $B$, $p$-Werte-Bereich für die Prüfung der Theorie (vgl. Tabelle Abb. 13). Normiert man jeweils die beiden axialen elektrischen Feldstärken in Abb. 15 auf den gleichen Anfangswert für $B=0$, so ändert sich $\Theta_{\exp }$, das Verhältnis der experimentellen Werte $E_{\| \mathbf{T}}$ zu $E_{\| \mathbf{L}}$, nicht sehr stark bei Variation des Entladungsstromes von $0,1 \mathrm{~A}$ bis $1 \mathrm{~A}$. Wir können deshalb die $\Theta_{\text {exp }}(B)$ für die zwei Stromwerte jeweils zu einem Mittelwert zusammenfassen, in ein $B, p$-Diagramm einzeichnen und mit den theoretischen, aus Abb. 13 mit Gl. (36) gewonnenen $\Phi$-Parametern vergleichen (Abb. 17). Aus dieser Abbildung ersieht man, daß zwar der Zusammenhang zwischen $B$ und $p$ für das experimentell bestimmte $\Theta_{\exp }$ mit dem für das berechnete $\Phi$ ungefähr übereinstimmt, daß aber $\Theta_{\exp }$ meistenteils über $\Phi$ liegt. Da Gl. (34) und Gl. (36) eine Abschätzung nach oben darstellen, läßt sich das experimentelle Ergebnis im Rahmen der oben ausgeführten Theorie nicht verstehen. Die Verluste im toroidalen Teil scheinen die zu erwartenden zu übertreffen.

Um dieses Ergebnis mit einem Gas mit höherer kritischer Magnetfeldstärke $B_{\mathrm{c}}$ zu testen, wurden die gleichen Experimente mit Xenon als Füllgas aus- 
$\mathbf{I}_{\mathrm{a}}=\mathbf{0 , 1} \mathrm{A}$

$\mathbf{I}_{\mathrm{a}}=\mathbf{1 A}$

$\mathbf{H}_{\mathbf{z}}$
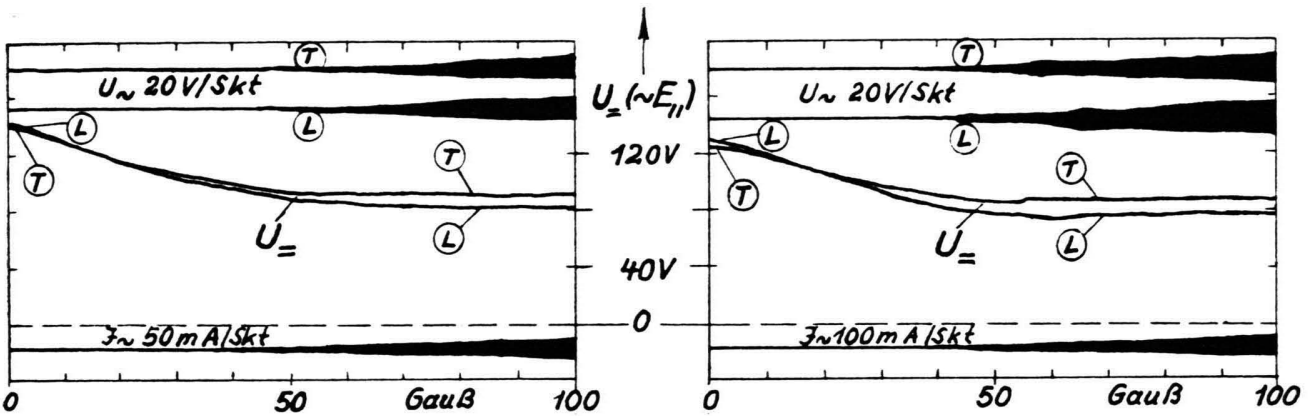

$10^{-2}$ Torr
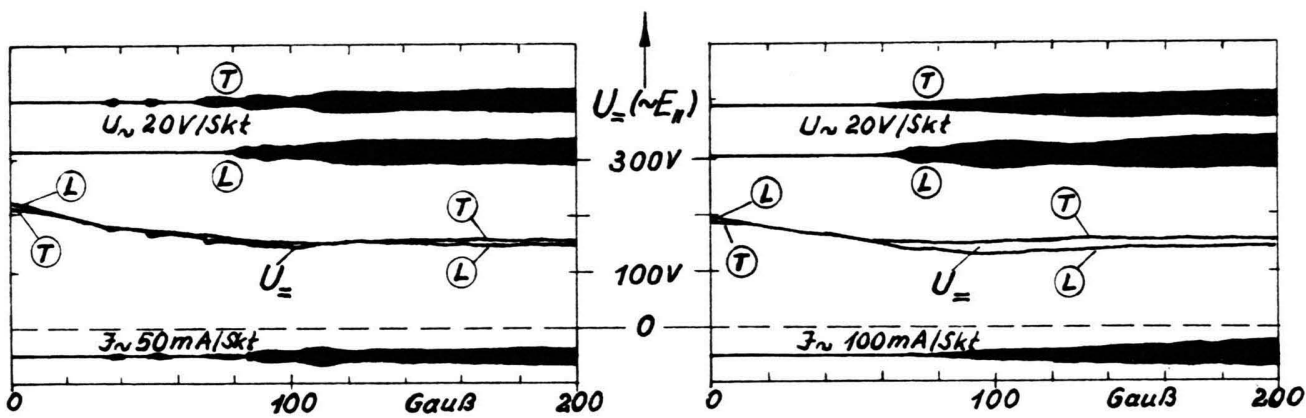

$3 \cdot 10^{-2}$ Torr
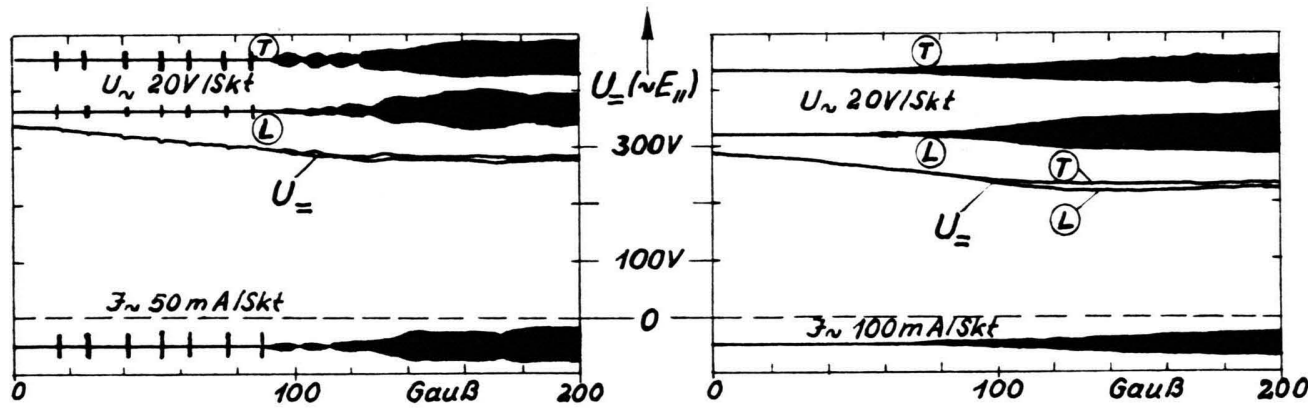

$10^{-1}$ Torr
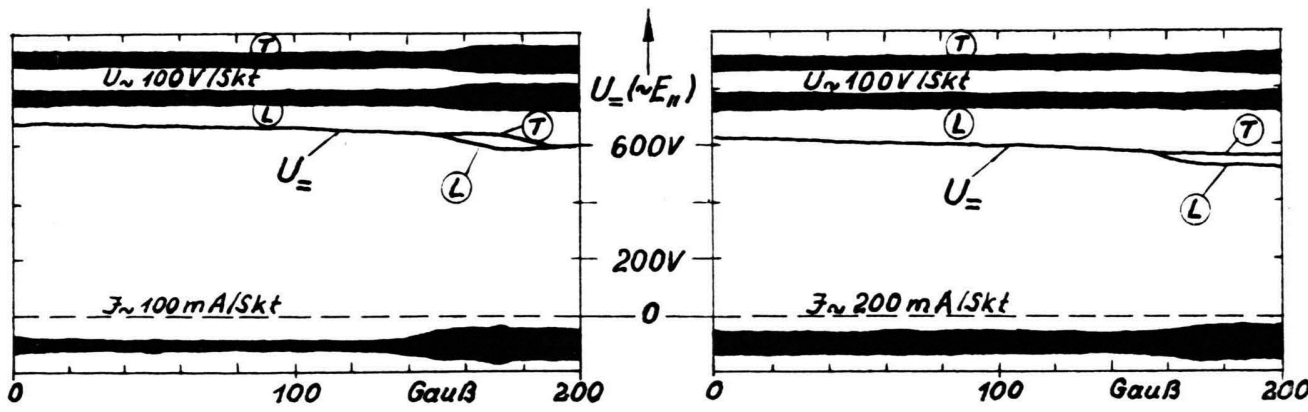

$3 \cdot 10^{-1}$ Torr

Abb. 14. $E_{\|}(B)$-Diagramme mit Rauschamplituden für Wasserstoff. 

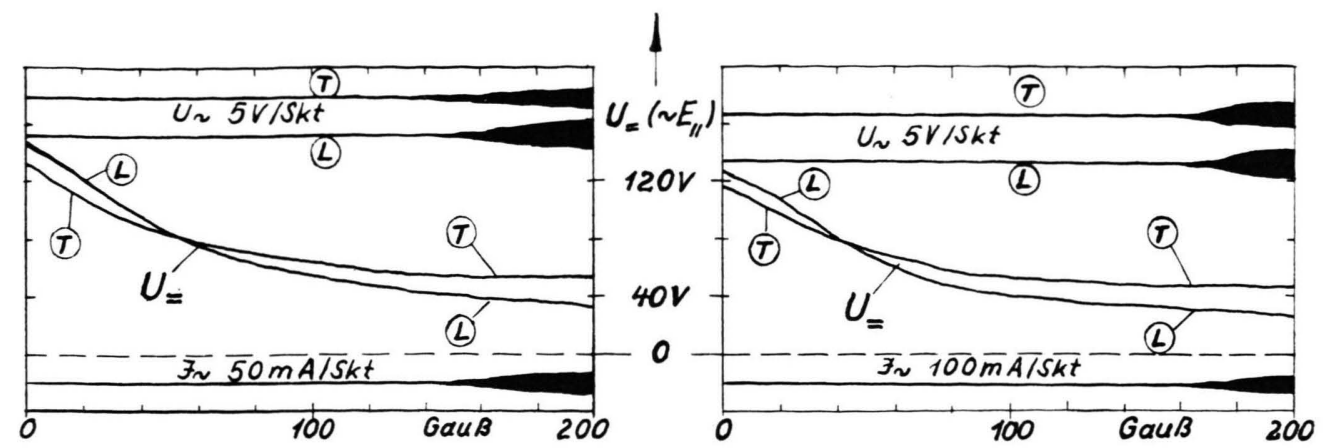

10-2 Torr
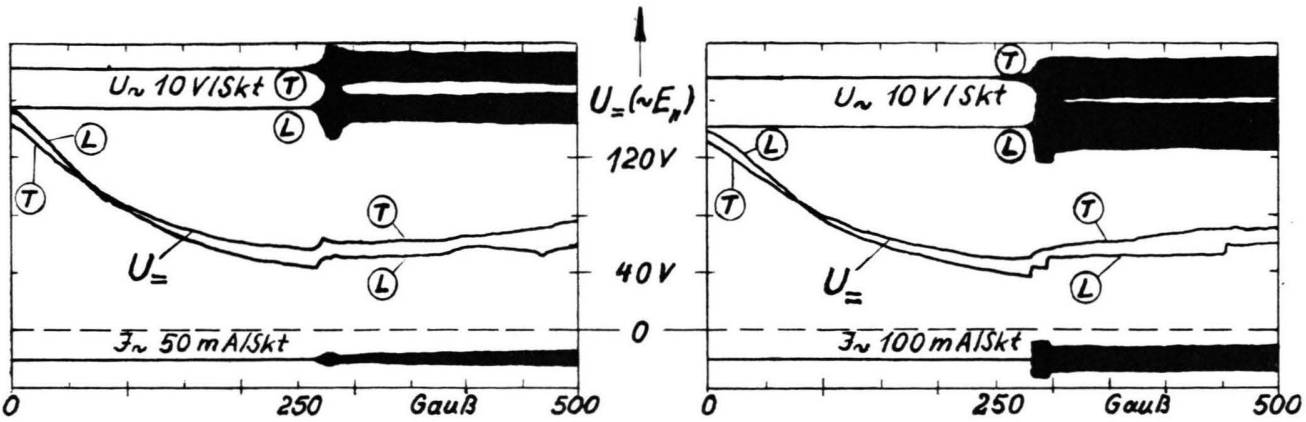

$3 \cdot 10^{-2}$ Torr
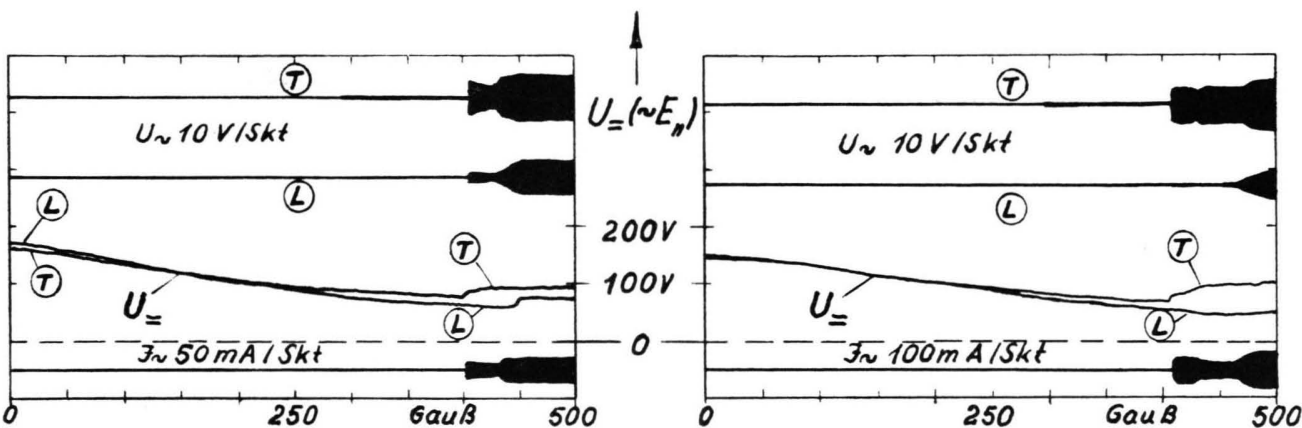

$10^{-1}$ Torr
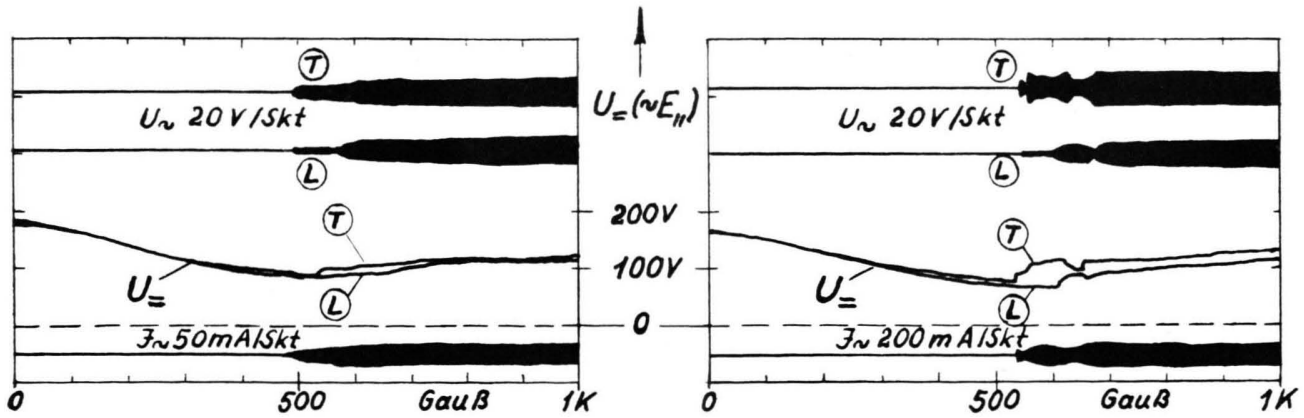

$3 \cdot 10^{-1}$ Torr
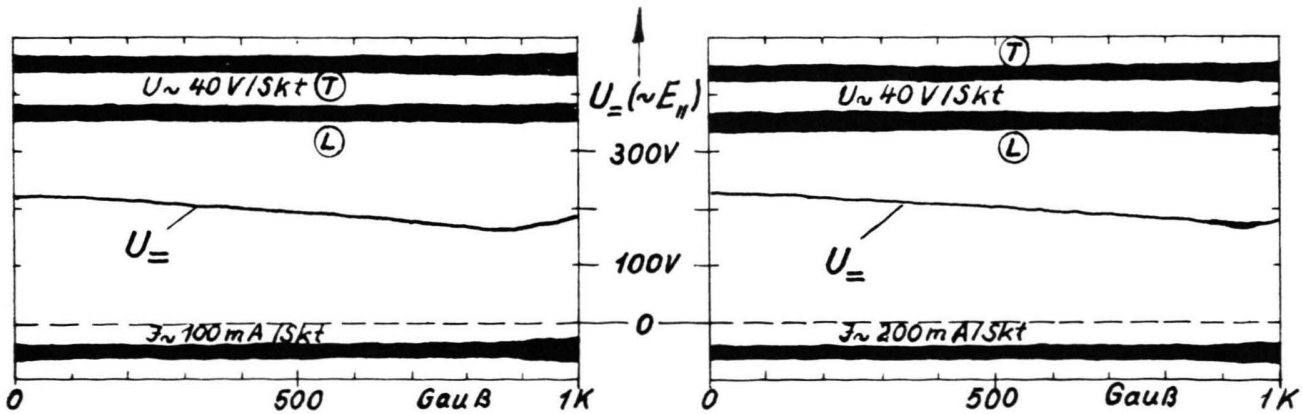

1 Torr

Abb. 15. $E_{\| \mid}(B)$-Diagramme mit Rauschamplituden für Helium. 


$$
I_{\mathrm{a}}=0,1 \mathrm{~A}
$$$$
I_{\mathrm{a}}=1 \mathrm{~A}
$$

$\mathrm{Xe}$
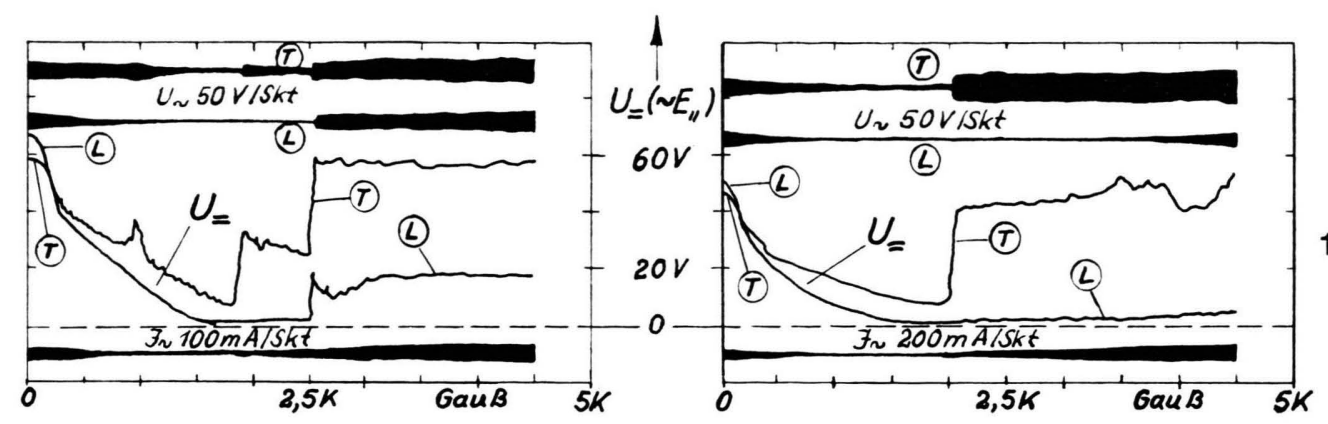

$10^{-2}$ Torr

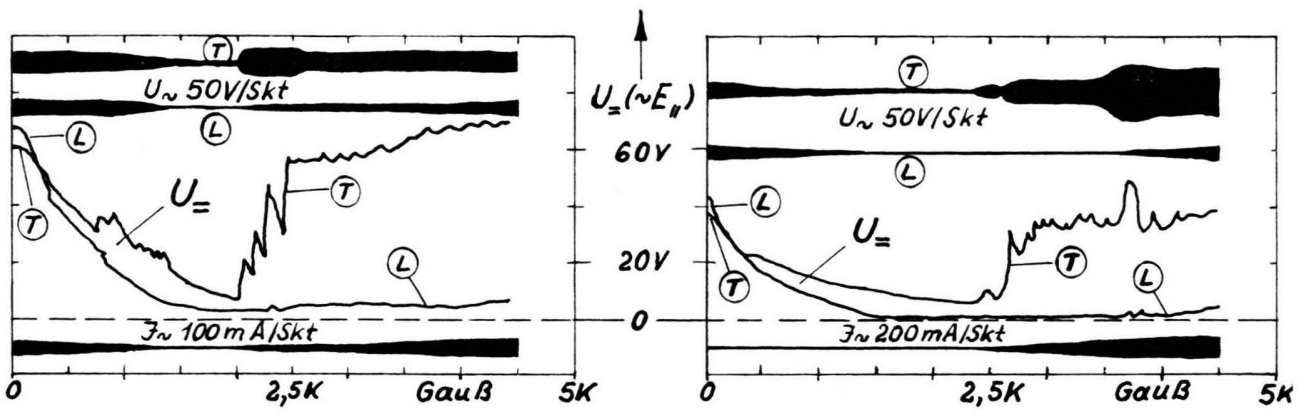

$3 \cdot 10^{-2}$ Torr

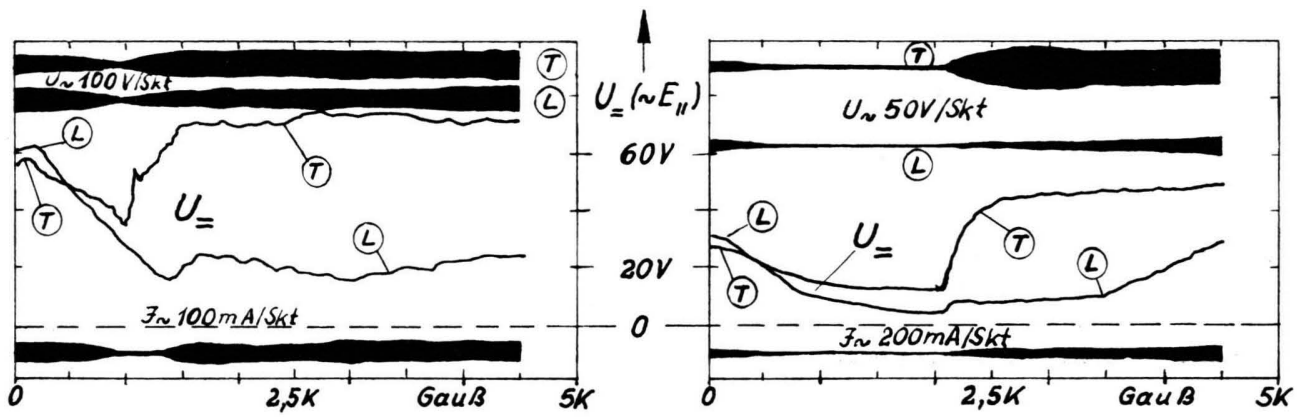

$1^{-1}$ Torr
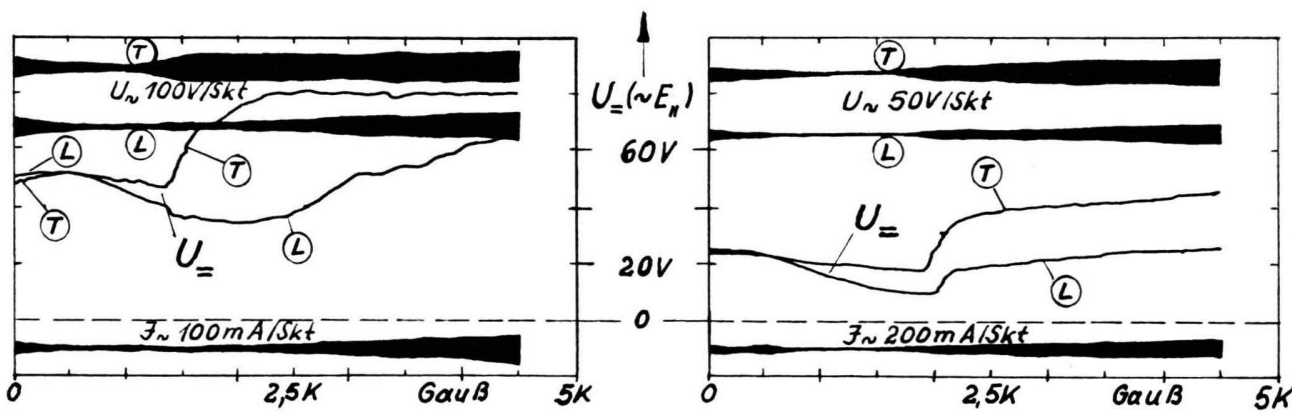

$3 \cdot 10^{-1}$ Torr

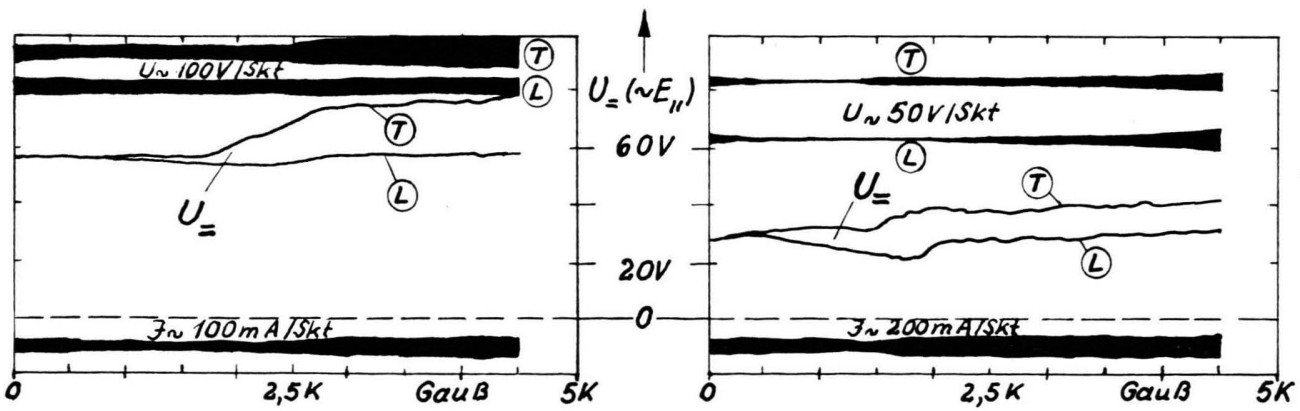

1 Torr

Abb. 16. $E_{\|}(B)$-Diagramme mit Rauschamplituden für Xenon. 


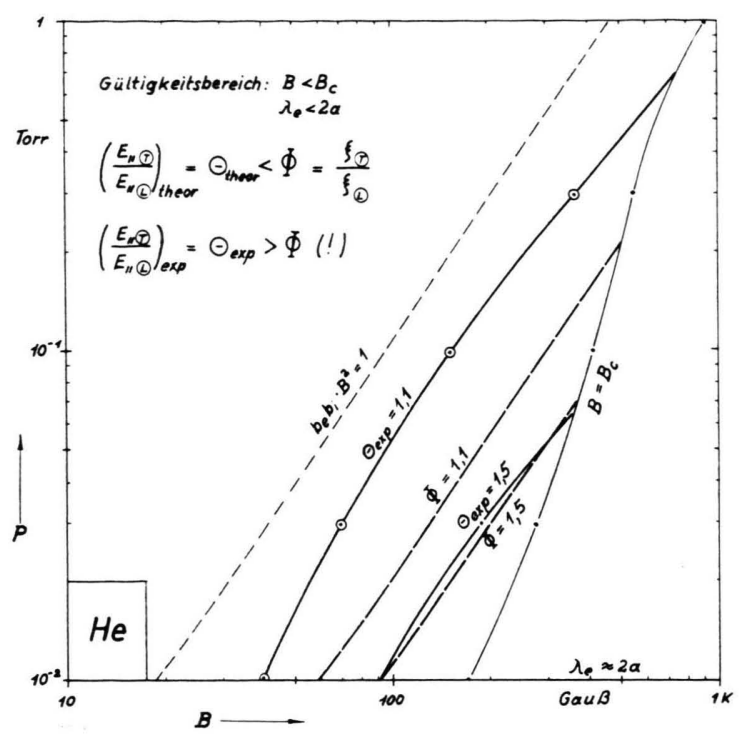

Abb. 17. $B, p$-Diagramm für das Verhältnis der Verluste im toroidalen und linearen Entladungsteil (Helium).

geführt (Abb. 16). Vergleicht man in diesem Fall $\Theta_{\exp }$ und $\Phi$ (Abb. 18; wegen der niedrigeren Brennspannung und dem daraus resultierenden stabileren Verhalten wurden nur die $I_{\mathrm{a}}=1$ A-Diagramme von Abb. 16 verwendet), so ergibt sich zwar wieder ungefähr der gleiche Gang mit $B$ und $p$, jedoch ist der Unterschied der Absolutwerte weitaus größer. Beispielsweise wird der Parameterwert $\Theta_{\exp }=1,1$ schon bei Magnetfeldstärken erreicht, die fast um eine

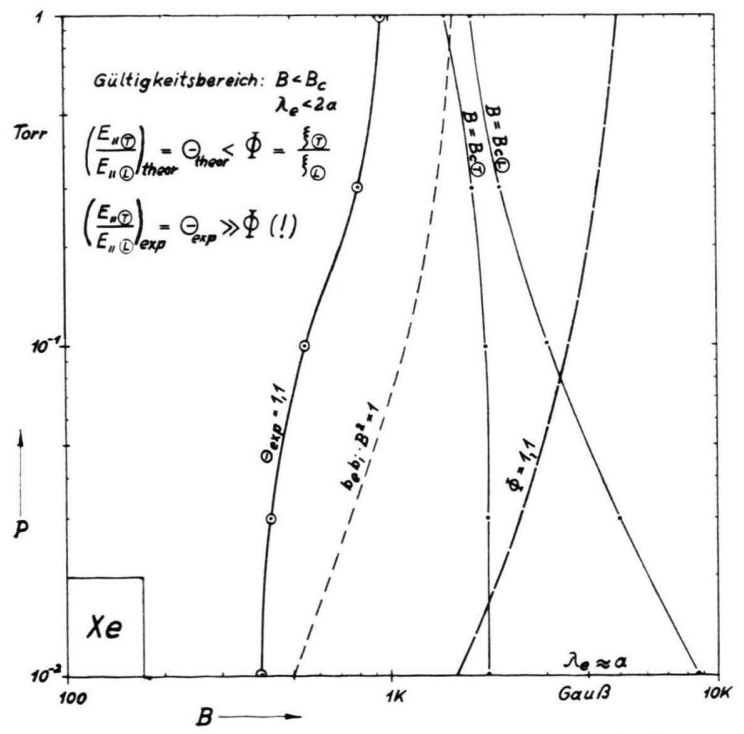

Abb. 18. $B, p$-Diagramm für das Verhältnis der Verluste im toroidalen und linearen Entladungsteil (Xenon).
Größenordnung unter der theoretischen Grenze für $\Phi=1,1$ liegen. Allerdings ist die Xenon-Entladung schon bei verschwindender Magnetfeldstärke bei allen Drucken leicht instabil (siehe Rauschamplituden Abb. 16). Da diese Instabilität offensichtlich mit steigendem Magnetfeld im linearen Teil stärker unterdrückt wird als im toroidalen, lassen sich bei Xenon nur qualitative Vergleiche zwischen Experiment und Theorie ziehen.

Zur nochmaligen Erweiterung des Gültigkeitsbereichs der Theorie durch stärkere Unterdrückung von Instabilitäten wurden alle Messungen wiederholt mit einer Anordnung, in der ein Teil der Ladungsträgerverluste durch eine Ionisierung mit HFEnergie kompensiert wird (vgl. B. II). Durch die daraus resultierende Erniedrigung der elektrischen Längsfeldstärke sollte der Einsatzpunkt der K-Instabilität nach höheren Magnetfeldstärken verschoben werden. Die dabei durch den Gleichrichtereffekt $^{10}$ an den Sonden entstehende Zusatzspannung, die bei gleichzeitiger Messung an drei Sonden auch durch Symmetrieren der Erdung der HF-Spannung nicht beseitigt werden kann, macht eine Korrektur der erhaltenen Kurven nötig. Dies geschieht mit Hilfe von Oszillogrammen, die mit der ausschließ. lich mit HF der gleichen Spannung betriebenen Entladung erhalten werden, deren Ergebnis an den Gleichstromkurven nachgeprüft werden muß (Beispiel: Abb. 19). Mit dieser Methode war es möglich, $B_{\mathrm{c}}$ bis um einen Faktor 8 zu erhöhen. Dadurch konnte nachgewiesen werden, daß das anomale $\Theta_{\exp }$ der Xenonentladung ohne zusätzliche HF-Ionisierung von der oben erwähnten Fluktuationsbewegung des Plasmas herrührt (Abb. 19, Xe, 10 $10^{-2}$ Torr, $1 \mathrm{~A})$; die Diskrepanz von $\Theta_{\text {exp }}$ und $\Theta_{\text {theor }}$ bei Helium änderte sich jedoch nicht merklich. Auch war es nicht möglich, das von $\mathrm{Gl}$. (31) vorausgesagte Minimum der Kurve $E_{\| \mathbf{T}}(B)$ im toroidalen Fall zu erreichen; ein allmähliches Wiederansteigen von $E_{\| \mathbf{T}}$ mit $B$, das in einigen mit zusätzlicher HF-Spannung erhaltenen Oszillogrammen beobachtet wurde, ließ sich mit Hilfe der Rauschamplitude $\left(U_{\sim}\right)$ von $E_{\| \mathbf{T}}$ in allen Fällen als Instabilität identifizieren.

\section{Diskussion}

Als Antwort auf die eingangs gestellte Frage haben Experiment und Theorie gezeigt, daß auch im toroidalen Teil der positiven Säule einer Gleichstromglimmentladung bei nicht zu starker Krüm. 

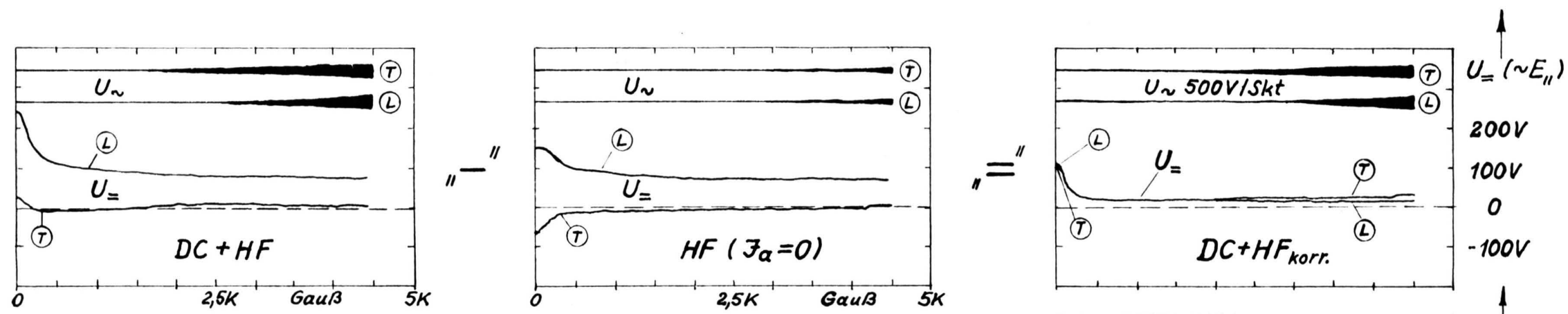

He $10^{-1}$ Torr, $I_{\mathrm{a}}=0,1 \mathrm{~A}$

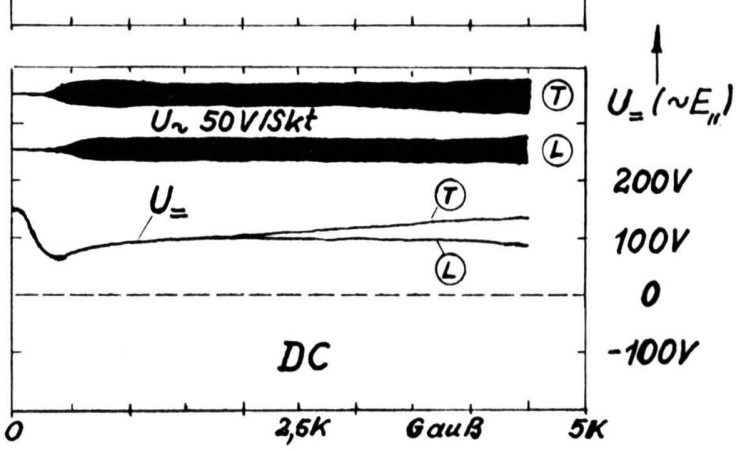

Xe $10^{-1}$ Torr, $I_{\mathrm{i}}=1 \mathrm{~A}$
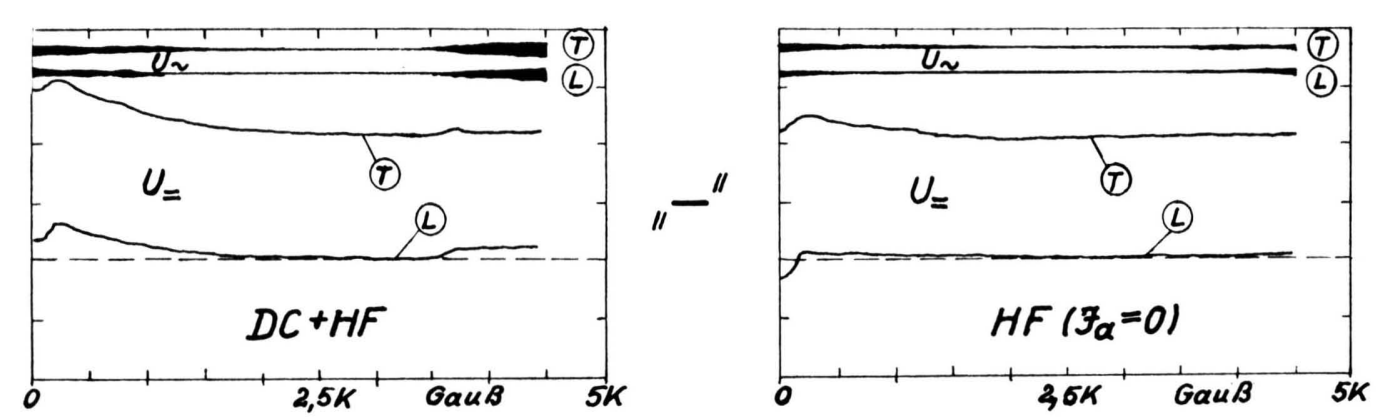

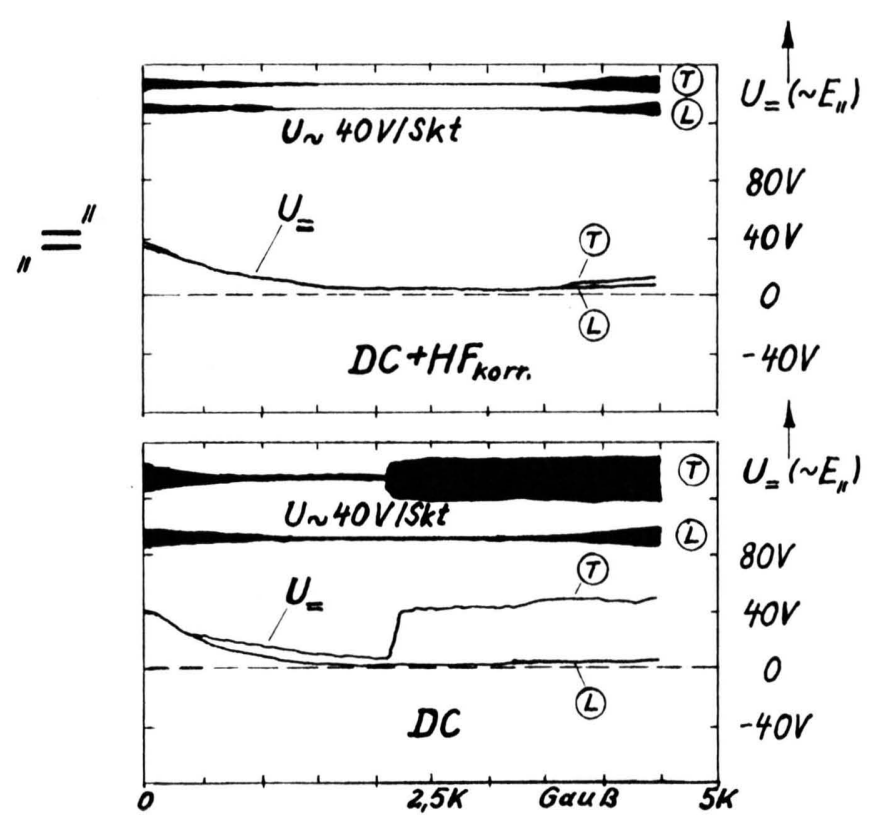

Abb. 19. $E_{||}(B)$-Diagramme mit zusätzlicher HF-Ionisierung. 
mung und nicht zu hohen Magnetfeldstärken ambipolare Diffusion $\left(B<B_{\mathrm{c}}\right)$ und Turbulenz $\left(B>B_{\mathrm{c}}\right)$ die beherrschenden Verlustmechanismen sind. Die aus der Torusdrift resultierenden Verluste sollten nach der oben abgeleiteten Theorie für $B<B_{\text {c }}$ nur eine untergeordnete Rolle spielen. Für die einem Vergleich mit der Theorie am besten zugängliche stabile Entladung in Helium ergab sich jedoch in der Nähe von $B_{c}$ eine Verlustrate, die außerhalb der Fehlergrenzen über dem theoretischen Wert liegt.

Folgende Erklärungsmöglichkeiten für die Diskrepanz zwischen Experiment und Theorie sollen deshalb diskutiert werden:

1. Fehler beim elektronischen Meßverfahren, evtl. Nullpunktsschwankungen.

2. Ungenügende Zentrierung der Entladungsröhre im toroidalen Magnetfeld.

3. Verwendung zu kleiner Werte der Beweglichkeiten.

4. Unzulässige Näherungen bei der Herleitung des Ausdrucks für die Ladungsträgererzeugungsrate im toroidalen Teil [Gl. (32) ].

5. Veränderung der Entladungsparameter bei Translation parallel zur Entladungsachse

a) Neutralgasdruck (infolge der Neutralgasdurchströmung),

b) Ladungsträgerdichte,

c) Radiales Ladungsträgerdichteprofil.

Bei diesen Erklärungsmöglichkeiten ist zu unterscheiden zwischen solchen, die das zu große $E_{\| \mathbf{T}}$ als echten durch die toroidale Krümmung verursachten Verlustmechanismus deuten (2., 3. und 4.) und denen, die nur eine Vortäuschung desselben behaupten (1. und 5.).

Gegen die Torusdrift als Ursache ist allgemein festzustellen, daß man im Falle höherer Verluste den Einsatzpunkt $B_{\mathrm{c}}$ der K-Instabilität zu höheren Magnetfeldstärken verschoben erwarten würde, da in diesem Fall nicht mehr so viel Energie in die Störung geliefert werden kann. Vergleicht man nämlich die theoretisch berechnete Torusdrift mit den stabilisierenden Driften und Diffusionen in einer helikalen Dichtestörung, so kommt man am rechten Rand des von uns betrachteten Gültigkeitsbereichs $\left(B=B_{\mathrm{c}}\right.$ in Abb. 17) zu vergleichbaren Werten.

Demgegenüber ist bei fast allen Diagrammen (Abb. 14 bis 16) zu beobachten [bei Xenon (Abb. 16 und 18) sogar ganz extrem], daß die K-Instabilität im toroidalen Teil schon bei niedrigeren Magnet- feldstärken einsetzt als im geraden. Die toroidale Krümmung könnte natürlich die bevorzugte Ausbildung einer Helix mit großer Wellen-Länge unterstützen; nachgewiesen wurde dieser Effekt jedoch noch nicht.

Gegen 2. kann weiterhin argumentiert werden, $\mathrm{da} ß$ der diskutierte Zentrierungstoleranzbereich von $3 \mathrm{~mm}$ (Glastorustoleranz) klein gegen $2 a=52 \mathrm{~mm}$ ist, so daß ein Durchtreten von Magnetfeldlinien durch die Wand die beobachteten Verluste mit an Sicherheit grenzender Wahrscheinlichkeit nicht verursachen kann.

Punkt 1. wurde mit Sicherheit ausgeschaltet durch Punkt-für-Punkt-Aufnahme einiger Kurven mit Hilfe eines Digitalröhrenvoltmeters direkt an den Sonden.

Eine Verwendung zu kleiner Beweglichkeiten (Punkt 3) ist nicht sehr wahrscheinlich, da die verwendeten Beweglichkeiten bei der Berechnung der $E_{\mid \mathbf{L}}(B)$-Kurve im zylindrischen Teil der Entladung - im Rahmen der Toleranz der Energiebilanz der positiven Säule - die Experimente richtig beschreiben (vgl. ${ }^{14}$ ), während zur Erklärung der experimentellen Torusverluste das Produkt $b_{\mathrm{i}} b_{\mathrm{e}}$ um den Faktor 5 erhöht werden müßte. Dies hätte bei hohen magnetischen Feldstärken ein merkbares Ansteigen von $E_{\| \mathbf{T}}(B)$ in der stabilen Entladung zur Folge, was jedoch experimentell nicht verifiziert werden konnte (Abb. 19, He, DC $+\mathrm{HF}$ ). Allerdings ist die Beeinflussung des radialen Dichteprofiles durch die zusätzliche HF-Spannung und die daraus resultierende Einwirkung auf den Verlustmechanismus nicht genau bekannt.

Der Einfluß der Näherungen vor allem bei der Berechnung der Torusdrift im Plasma mit Neutralgas ist noch nicht völlig zu übersehen. Im Falle der Gültigkeit von Punkt 4 dürfte der Anstieg von $\xi_{\mathbf{T}}-\xi_{\mathbf{L}}[$ [Gl. (31) ] jedoch nicht mehr quadratisch in $B$ sein, um mit dem schwachen Anstieg von $E_{\|}$in Abb. 19 ( He, DC $\left.+\mathrm{HF}_{\text {korr }}\right)$ bei hohen Magnetfeldstärken konsistent zu bleiben. Ob dies der Fall ist, läßt sich erst durch Messung der die Torusdrift erzeugenden Radialfelder beantworten.

5. a) wurde dadurch gegenstandslos, daß bei Neutralgasströmung in die entgegengesetzte Richtung die gleichen Ergebnisse erhalten wurden. 5.b) ist auf Grund der theoretisch und experimentell bekannten Struktur der positiven Säule (Abb.2) nicht wahrscheinlich. 5.c) könnte ein konsistentes Bild der Experimente liefern: Durch die am Spulenende divergierenden Kraftlinien des magnetischen Feldes 
werden die aus der Kathode ausgetretenen Elektronen zur Entladungsachse hin konzentriert. Damit wäre eine Abweichung des Dichteprofils von der Besselschen Form Gl. (13) verbunden, die sich entlang der Entladung bis zur Anode wieder ausgleicht. Das schärfere Dichteprofil hätte niedrigere Dichtegradienten am Rand und damit geringere Verluste im geraden Teil zur Folge; die daraus resultierende niedrigere elektrische Längsfeldstärke würde zu einem höheren $B_{\text {c }}$ gegenüber dem der Anode näheren Teil der Entladung führen.

Wir sehen, daß dieses Bild die experimentellen Resultate qualitativ erklären könnte. Die endgültige Entscheidung zwischen 3., 4. und 5.c) kann erst eine verfeinerte theoretische und experimentelle Ana- lyse der zur Torusdrift führenden elektrischen Radialfelder und Dichteprofile, eine Erweiterung des Gültigkeitsbereiches der Theorie durch weitere Erhöhung von $B_{\text {c }}$ sowie eine Untersuchung der Verluste bei Richtungsumkehr der longitudinalen elektrischen Feldstärke bringen, was einer späteren Arbeit vorbehalten sein soll.

Ich danke Herrn Prof. Dr. Heisenberg und Herrn Prof. Dr. Biermann für die Möglichkeit, die vorstehende Arbeit am Max-Planck-Institut für Physik und Astrophysik auszuführen. Mein Dank gilt auch Herrn Dr. Eckhartt, Herrn Dr. v. Gierke und Herrn Dr. Grieger, sowie anderen Mitarbeitern des Institutes für ihre Unterstützung. Fräulein KARGER und Herrn $E_{\text {RTL }}$ bin ich für ihre Mithilfe bei komplizierten Experimenten dankbar.

\title{
Investigation of a Caesium Plasma Diode Using an Electron Beam Probing Technique ${ }^{1,2}$
}

\author{
Werner OtT \\ Institut für Plasmaphysik GmbH, Garching bei München
}

(Z. Naturforschg. 22 a, 1057-1067 [1967] ; received 23 March 1967)

\begin{abstract}
The plasma in a plane caesium diode with a hot emitter and a cold collector was investigated experimentally with a ribbon-shaped electron beam. The ribbon beam is projected through the diode at an angle of 45 degrees to its axis and allowed to strike a fluorescent screen. Variations in the axial electric field of the diode cause the ribbon beam to be distorted. The image of the distorted beam as seen on the fluorescent screen then constitutes a plot of the axial electric field along the axis of the diode.

The field plots so obtained are compared with a theory in which the collisions of the charge carriers are neglected. By means of this comparison it is possible to evaluate the neutralization parameter, the plasma density, and an average drift energy of the charge carriers.

The results show that the theory correctly describes the different modes of the potential distribution and especially the transitions between modes of operation as long as the diode is free of oscillations.

The stability of the different possible static potential distributions was also investigated. It was found experimentally that the system is unstable if the electron emission is less than the ion emission and the collector potential is positive.
\end{abstract}

Since the ionization of atoms of low ionization energy on hot surfaces with high work function was discovered by Langmuir and Kingdon ${ }^{3}$ in 1925 , widespread use has been made of contact ionization.

Plasmas produced by contact ionization can be maintained without currents. This is a great advan-

1 Auszug aus der von der Fakultät für allgemeine Wissenschaften der Technischen Hochschule München genehmig. ten Dissertation über „Untersuchung der CäsiumplasmaDiode mit einer neuen Elektronenstrahlsonden-Technik" des Verfassers. tage because currents cause many kinds of instabilities. Such plasmas are therefore useful for investigating the conditions necessary for thermonuclear fusion. The fact that a deuterium plasma with a temperature of more than $100000{ }^{\circ} \mathrm{K}$ can be simulated by a caesium plasma (on account of the large

\footnotetext{
2 This work was performed under the terms of the agreement on association between the Institut für Plasmaphysik and EURATOM.

3 I. Langmuir and K. H. Kingdon, Proc. Roy. Soc. London A 107, 61 [1925].
} 